\title{
"Kinetic NMR-Titration": Including Chemical Shift Information in the Kinetic Analysis of Supramolecular Reaction Systems such as Organic Replicators
}

Insa Stahl, Günter von Kiedrowski ${ }^{*}$

\author{
Ruhr-Universität Bochum, Lehrstuhl für Organische Chemie I, Universitätsstrasse 150, 44780 Bochum, Germany
}

kiedro@rub.de

\section{Supporting Information}

\section{Example SimFit command file (input file)}

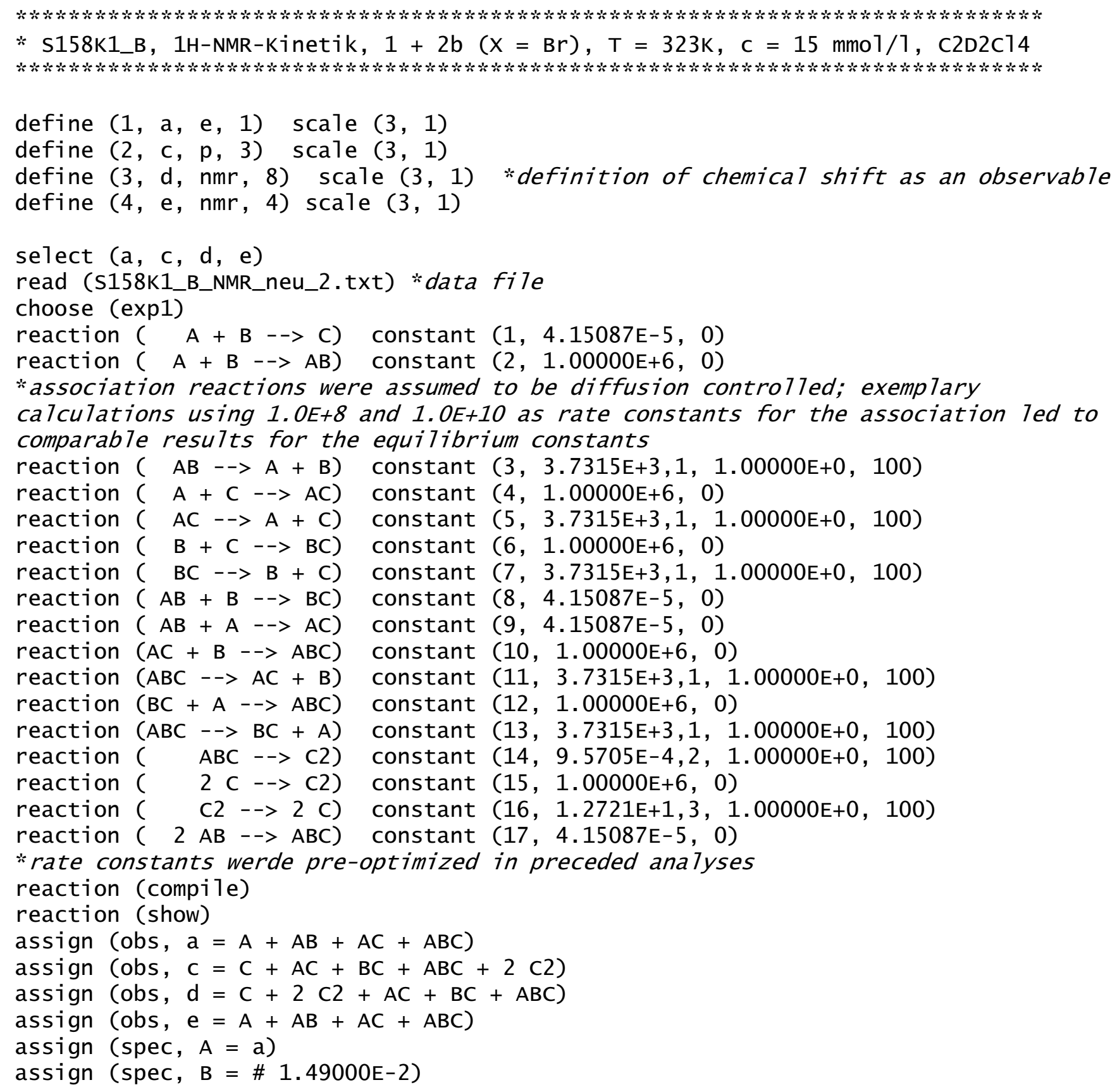


assign $($ spec, $c=\# 0.00000 \mathrm{E}+0)$

czero (4, a, 1, 1, 1.05)

"chemical shift iteration for the species according to the reaction mode 7

de1ta (d, C, 8.2597E+0, 5.00000E+0, 7.00000E+0, 9.00000E+0)

delta (d, AC, 8.2597E+0, 5.00000E+0, 7.00000E+0, 9.00000E+0)

delta (d, BC, 8.0161E+0, 7.00000E+0, 7.00000E+0, 9.00000E+0)

de1ta (d, ABC, 8.2631E+0, 8.00000E+0, 7.00000E+0, 9.00000E+0)

de1ta (d, C2, 8.2968E+0, 6.00000E+0, 7.00000E+0, 9.00000E+0)

delta (e, A, 8.0135E+0, 9.00000E+0, 7.00000E+0,9.00000E+0)

delta (e, $A B, 8.2635 E+0,1.00000 E+1,7.00000 E+0,9.00000 E+0)$

delta (e, $A C, 8.2635 \mathrm{E}+0,1.00000 \mathrm{E}+1,7.00000 \mathrm{E}+0,9.00000 \mathrm{E}+0$ )

delta (e,ABC, 8.1735E+0, 1.10000E+1, 7.00000E+0, 9.00000E+0)

time (h)

conc (mM)

win $(0,70,10,0,-1,16,2,0)$

nmrwin $(0,70,10,0,8.05,8.30,0.1,0) *$ set

$\operatorname{dim}(11)$

int (stiff, $1 \mathrm{e}-7,32,0.05,500,1500$ )

plot (spec)

simplex (plot)

opar $(1 e+8)$

newton (plot)

plot (spec)

plot (resi)

make

\section{SimFit plot results}
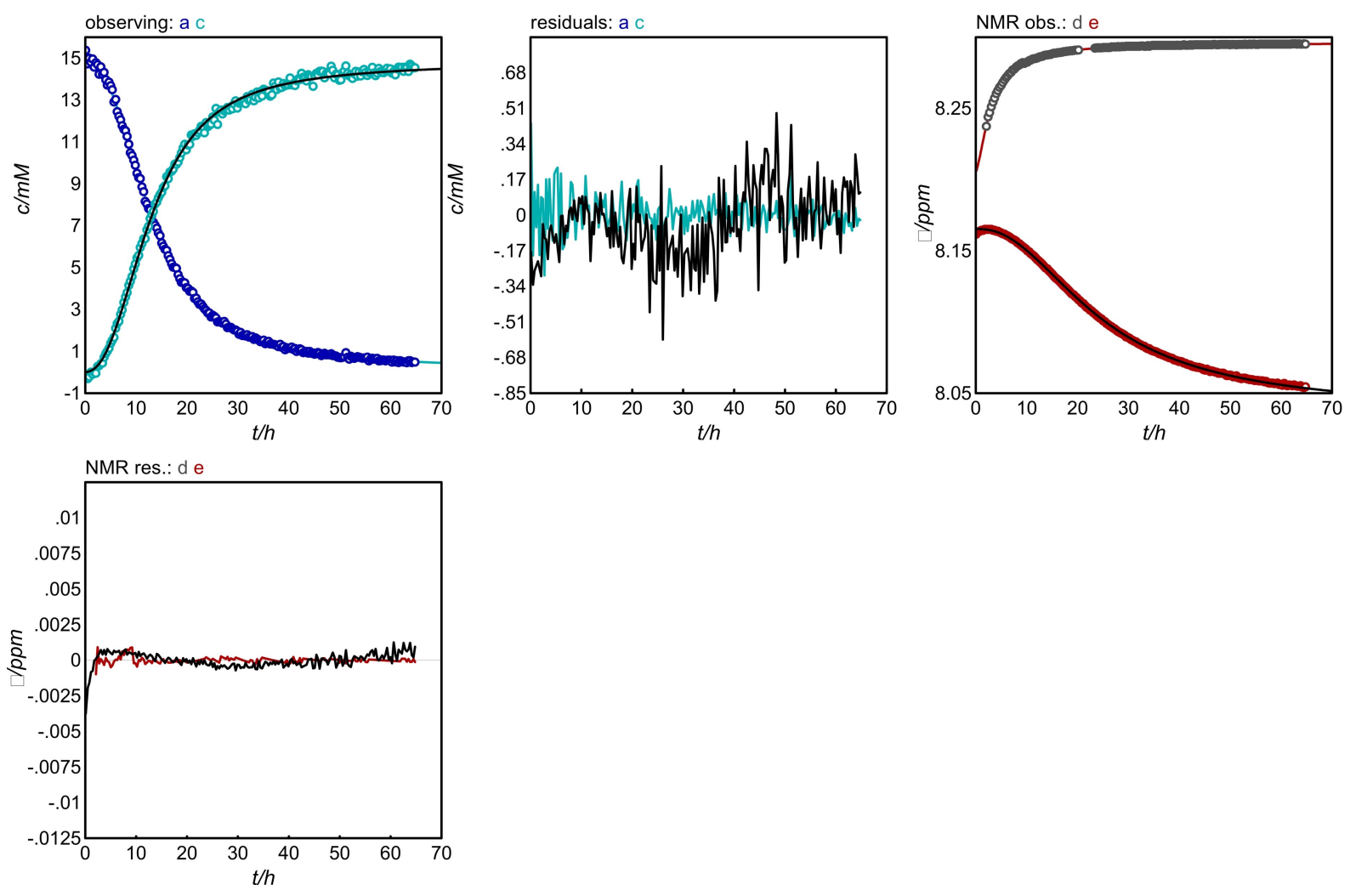
$* * * * * * * * * * * * * * * * * * * * * * * * * * * * * * * * * * * * * * * * * * * * * * * * * * * * * * * * * * * * * * * * * * * * * * * * *$ * simfit (PB/DLL6) 32-bit (10-Feb-2003) (C) 1989-2001 G. V. Kiedrowski *

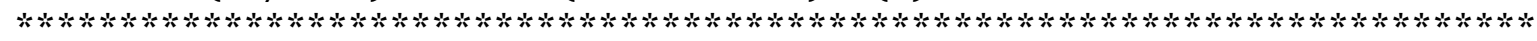

This version of Simfit comes with 2 GB variable space.

SF32.INI is currently set to:

63 species, 63 reactions, 24 iteratable rate constants, 32 observables, 2 files, 10 experiments/file,256 reaction times/experiment.

$\operatorname{DEFINE}(1, \mathrm{a}, \mathrm{e}, 1)$

$\operatorname{SCALE}(3,1)$

$\mathrm{a}: \mathrm{K} 1 * \mathrm{FE}$

$\operatorname{DEFINE}(2, c, p, 3)$

$\operatorname{SCALE}(3,1)$

C: $\mathrm{K} 1 * \mathrm{FE}$

$\operatorname{DEFINE}(3, \mathrm{~d}, \mathrm{nmr}, 8)$

$\operatorname{SCALE}(3,1)$

$\mathrm{d}: \mathrm{K} 1 * \mathrm{FE}$

DEFINE $(4, \mathrm{e}, \mathrm{nmr}, 4)$

$\operatorname{SCALE}(3,1)$

e: $\mathrm{K} 1 * \mathrm{FE}$

SELECT $(a, c, d, e)$

READ (s158k1_b_nmr_neu_2.txt)

The following observables found in s158k1_b_nmr_neu_2.txt are DEFINEd:

a

c

d

e

Unit of time is not defined in data file.

simfit assumes minutes as unit of time.

CHOOSE (exp1)

REACTION $(a+b-->c)$

CONSTANT $(1,4.15087 \mathrm{e}-5,0)$

REACTION $(a+b-->a b)$

CONSTANT $(2,1.00000 \mathrm{e}+6,0)$

REACTION ( $a b-->a+b)$

CONSTANT $(3,3.7315 \mathrm{e}+3,1,1.00000 \mathrm{e}+0,100)$

REACTION (a + c--> ac)

CONSTANT $(4,1.00000 \mathrm{e}+6,0)$ 


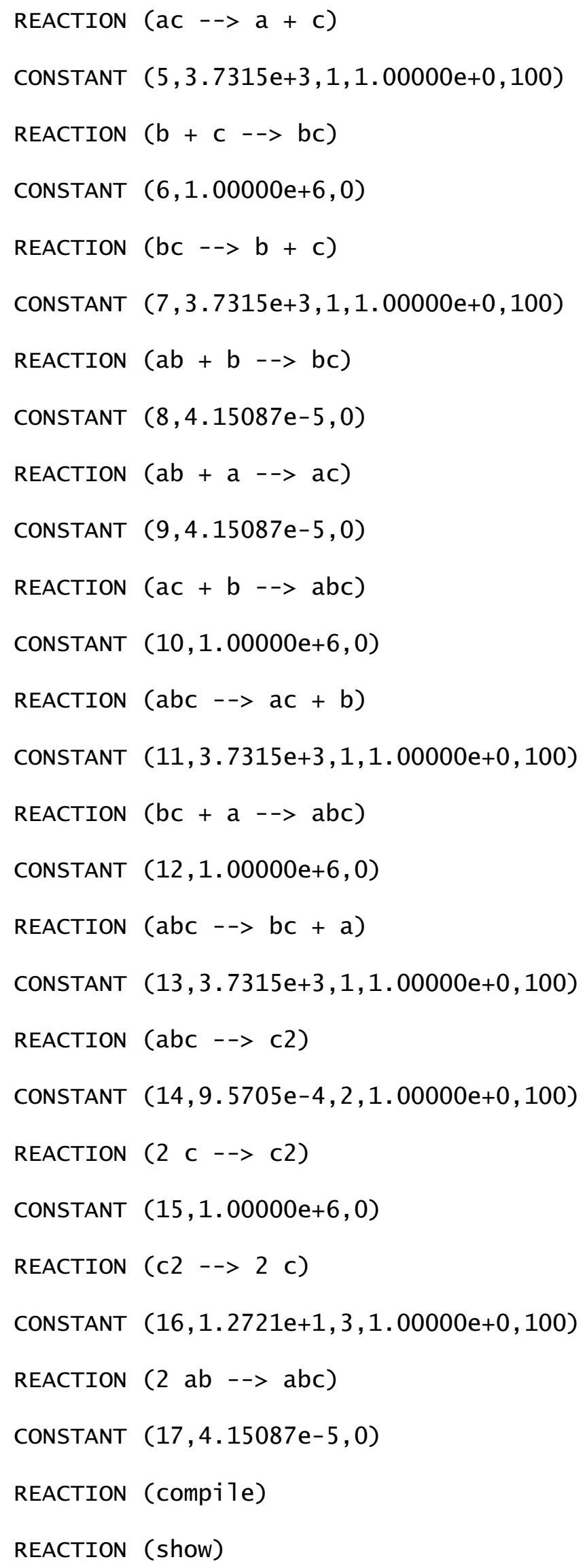


Reactions:

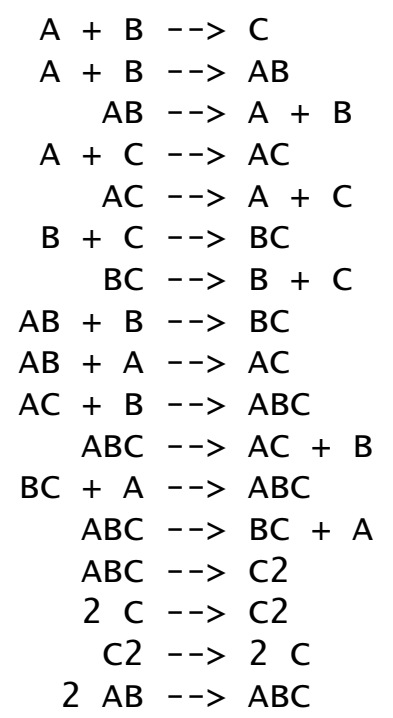

Species found: $A$ A $\quad C \quad A B$ AC $B C \quad A B C \quad C 2$

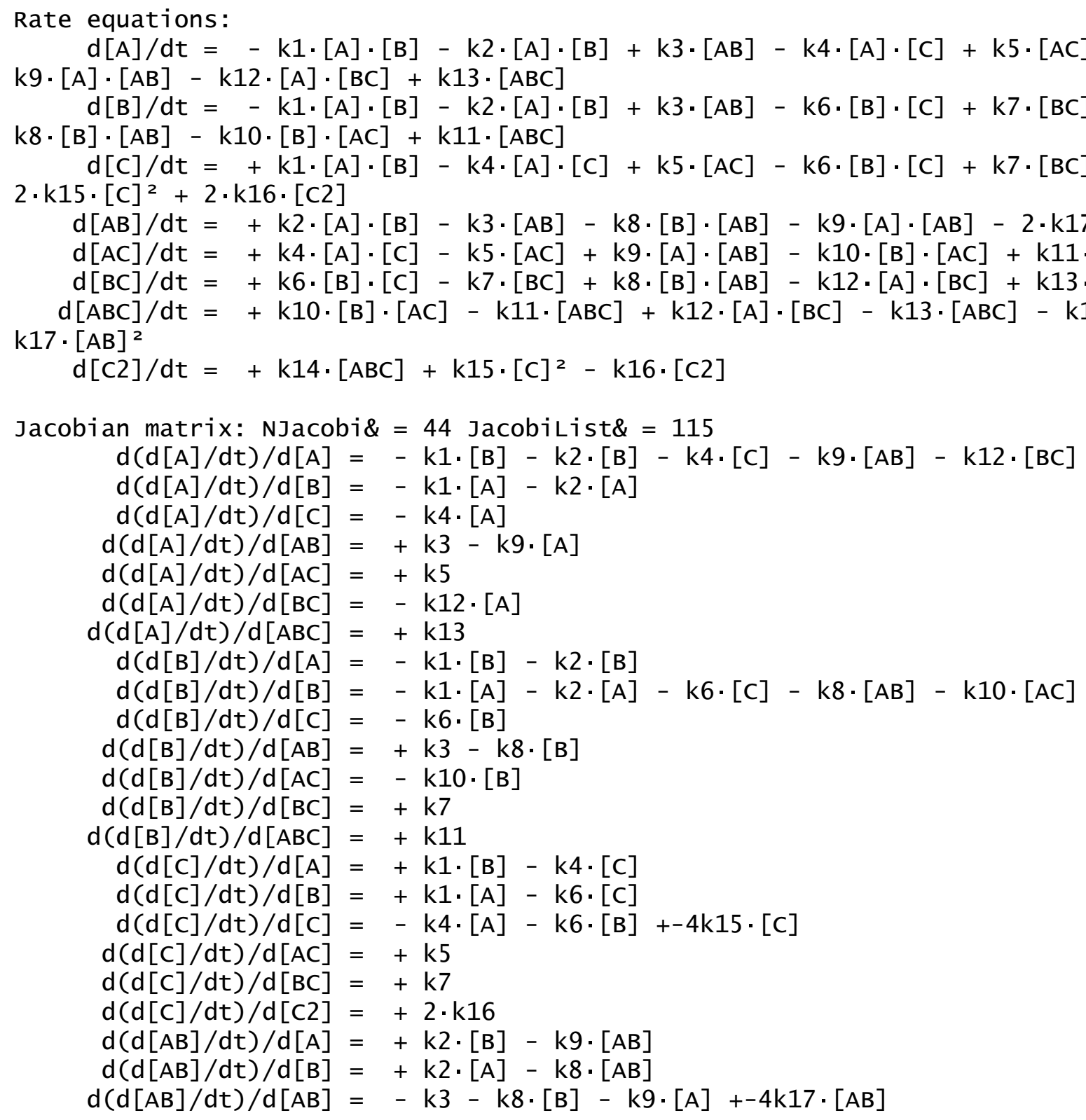




$$
\begin{aligned}
& \mathrm{d}(\mathrm{d}[\mathrm{AC}] / \mathrm{dt}) / \mathrm{d}[\mathrm{A}]=+\mathrm{k} 4 \cdot[\mathrm{C}]+\mathrm{k} 9 \cdot[\mathrm{AB}] \\
& d(d[A C] / d t) / d[B]=-k 10 \cdot[A C] \\
& d(d[A C] / d t) / d[C]=+k 4 \cdot[A] \\
& d(d[A C] / d t) / d[A B]=+k 9 \cdot[A] \\
& \mathrm{d}(\mathrm{d}[A C] / \mathrm{dt}) / \mathrm{d}[A C]=-\mathrm{k} 5-\mathrm{k} 10 \cdot[\mathrm{B}] \\
& d(d[A C] / d t) / d[A B C]=+k 11 \\
& \mathrm{~d}(\mathrm{~d}[\mathrm{BC}] / \mathrm{dt}) / \mathrm{d}[\mathrm{A}]=-\mathrm{k} 12 \cdot[\mathrm{BC}] \\
& \mathrm{d}(\mathrm{d}[\mathrm{BC}] / \mathrm{dt}) / \mathrm{d}[\mathrm{B}]=+\mathrm{k} 6 \cdot[\mathrm{C}]+\mathrm{k} 8 \cdot[\mathrm{AB}] \\
& \mathrm{d}(\mathrm{d}[\mathrm{BC}] / \mathrm{dt}) / \mathrm{d}[\mathrm{C}]=+\mathrm{k} 6 \cdot[\mathrm{B}] \\
& d(d[B C] / d t) / d[A B]=+k 8 \cdot[B] \\
& \mathrm{d}(\mathrm{d}[\mathrm{BC}] / \mathrm{dt}) / \mathrm{d}[\mathrm{BC}]=-\mathrm{k} 7-\mathrm{k} 12 \cdot[\mathrm{A}] \\
& \mathrm{d}(\mathrm{d}[\mathrm{BC}] / \mathrm{dt}) / \mathrm{d}[\mathrm{ABC}]=+\mathrm{k} 13 \\
& d(d[A B C] / d t) / d[A]=+k 12 \cdot[B C] \\
& \mathrm{d}(\mathrm{d}[\mathrm{ABC}] / \mathrm{dt}) / \mathrm{d}[\mathrm{B}]=+\mathrm{k} 10 \cdot[\mathrm{AC}] \\
& \mathrm{d}(\mathrm{d}[\mathrm{ABC}] / \mathrm{dt}) / \mathrm{d}[\mathrm{AB}]=+2 \cdot \mathrm{k} 17 \cdot[\mathrm{AB}] \\
& d(d[A B C] / d t) / d[A C]=+k 10 \cdot[B] \\
& d(d[A B C] / d t) / d[B C]=+k 12 \cdot[A] \\
& \mathrm{d}(\mathrm{d}[\mathrm{ABC}] / \mathrm{dt}) / \mathrm{d}[\mathrm{ABC}]=-\mathrm{k} 11-\mathrm{k} 13-\mathrm{k} 14 \\
& \mathrm{~d}(\mathrm{~d}[\mathrm{C} 2] / \mathrm{dt}) / \mathrm{d}[\mathrm{C}]=+2 \cdot \mathrm{k} 15 \cdot[\mathrm{C}] \\
& \mathrm{d}(\mathrm{d}[\mathrm{C} 2] / \mathrm{dt}) / \mathrm{d}[\mathrm{ABC}]=+\mathrm{k} 14 \\
& d(d[C 2] / d t) / d[C 2]=-k 16
\end{aligned}
$$


WIN $(0,70,10,0,-1,16,2,0)$

NMRWIN $(0,70,10,0,8.05,8.30,0.1,0)$

DIM (11)

INT (stiff, 1e-7, 32, $0.05,500,1500)$

PLOT (spec)

SIMPLEX (plot)

$\mathrm{k} 1 \_\mathrm{k} 2$

$\mathrm{k} 8 \longrightarrow \mathrm{k} 9$

initial values:

3.7315E+3 9.5705E-4

8. $2631 \mathrm{E}+0$

8. $0135 \mathrm{E}+0$

1. $2721 \mathrm{E}+1$

8. $2635 \mathrm{E}+0$

k3

k10

k4

$k 11$

$\mathrm{k} 5$

R.M.S. [\%]

k6

1. $5370 \mathrm{E}-2$

$8.1735 \mathrm{E}+0$

$8.2597 \mathrm{E}+0$

8. $2968 \mathrm{E}+0$

8. $0161 \mathrm{E}+0$

8.5788
$8.2334 \mathrm{E}+0$

8.2863

$8.1882 \mathrm{E}+0$

8. $0150 \mathrm{E}+0$

$8.2678 \mathrm{E}+0$

$8.2678 \mathrm{E}+0$

$8.3675 \mathrm{E}+0$

1. $3331 \mathrm{E}+1$

$8.2695 \mathrm{E}+0$

1. $5162 \mathrm{E}-2$

8. $1784 \mathrm{E}+0$

8. $0157 \mathrm{E}+0$

1. $3260 \mathrm{E}+1$

$8.2646 \mathrm{E}+0$

1. $5210 \mathrm{E}-2$

4.0129E+3 9.5366E-4

8. $2533 \mathrm{E}+0 \quad 8.0151 \mathrm{E}+0$

1. $3544 \mathrm{E}+1$

8. 3194E+0

$3.7971 \mathrm{E}+3$

8. $2273 \mathrm{E}+0$

9. $4845 \mathrm{E}-4$

8. $0144 \mathrm{E}+0$

8. $2630 \mathrm{E}+0$

1. $5122 \mathrm{E}-2$

$8.3860 \mathrm{E}+0$

$3.9491 E+3$

9. $5658 \mathrm{E}-4$

1. $3542 \mathrm{E}+1$

8. $2613 \mathrm{E}+0$

$1.4988 \mathrm{E}-2$

8. $2696 \mathrm{E}+0$

8. $0135 \mathrm{E}+0$

$3.8898 \mathrm{E}+3 \quad 9.6925 \mathrm{E}-4$

1. $3561 \mathrm{E}+1$

8. $2668 \mathrm{E}+0$

8. $2739 \mathrm{E}+0$

5.3682

8. $2612 \mathrm{E}+0$

4.8380

8. $2636 \mathrm{E}+0$

3.5345

8. 2427E+0

2.9070

$8.2523 \mathrm{E}+0$

8. $0147 \mathrm{E}+0$

$8.2645 \mathrm{E}+0$

1. 5041E-2

8. $2691 \mathrm{E}+0$

2. 5051

$3.8956 \mathrm{E}+3 \quad 9.6475 \mathrm{E}-4$

8. $2288 \mathrm{E}+0$

8. $0144 \mathrm{E}+0$

1. $3597 \mathrm{E}+1$

8. $2641 \mathrm{E}+0$

1. $4985 \mathrm{E}-2$

$8.2773 \mathrm{E}+0$

8. $2698 \mathrm{E}+0$

2.4927

$3.7798 \mathrm{E}+3$

8. $2457 \mathrm{E}+0$

$9.7600 \mathrm{E}-4$

1. $3678 \mathrm{E}+1$

1. $4938 \mathrm{E}-2$

8. $0137 \mathrm{E}+0$

$8.2608 \mathrm{E}+0$

$8.2734 \mathrm{E}+0$

8. 2569E+0

2.0748

$3.8822 \mathrm{E}+3 \quad 9.6459 \mathrm{E}-4$

1. $3535 \mathrm{E}+1$

$1.4958 \mathrm{E}-2$

$8.2540 \mathrm{E}+0$

8. $0139 \mathrm{E}+0$

$8.2625 \mathrm{E}+0$

8. $2789 \mathrm{E}+0$

8. 2637E+0

2.0225

$3.8029 \mathrm{E}+3 \quad 9.6816 \mathrm{E}-4$

8. $2623 \mathrm{E}+0$

8. $0142 \mathrm{E}+0$

1. $3618 \mathrm{E}+1$

$1.4933 \mathrm{E}-2$

8. $2624 \mathrm{E}+0$

8. $2720 \mathrm{E}+0$

$8.2604 \mathrm{E}+0$

1.9508

$3.8566 \mathrm{E}+3 \quad 9.6101 \mathrm{E}-4$

8. $2676 \mathrm{E}+0$

8. $0135 \mathrm{E}+0$

1. 3587E+1

8. $2613 \mathrm{E}+0$

1. $4965 \mathrm{E}-2$

8. $2499 \mathrm{E}+0$

8. $2611 \mathrm{E}+0$

1.8185

$3.8581 \mathrm{E}+3$

8. $2680 \mathrm{E}+0$

9.7683E-4

1. $3608 \mathrm{E}+1$

1. 4891E-2

8. $0136 \mathrm{E}+0$

8. $2619 \mathrm{E}+0$

8. $2224 \mathrm{E}+0$

8. $2598 \mathrm{E}+0$

1.5750

3. $8108 \mathrm{E}+3$

8. $2506 \mathrm{E}+0$

9. $7223 \mathrm{E}-4$

1. $3630 \mathrm{E}+1$

1. $4950 \mathrm{E}-2$

8. $2616 \mathrm{E}+0$

8. $2724 \mathrm{E}+0$
8. $2600 \mathrm{E}+0$

1.5508 8. $0138 \mathrm{E}+0$
8. 2968E+0

$8.0785 \mathrm{E}+0$

8. 2972E+0

8. 0932E+0

8. 2986E+0

$8.0535 \mathrm{E}+0$

8. 2964E+0

8. 0514E+0

8. 2966E+0

8. $0261 E+0$

8. 2967E+0

$8.0559 \mathrm{E}+0$

8. $2970 \mathrm{E}+0$

8. $0492 \mathrm{E}+0$

8. 2959E+0

8. $0510 \mathrm{E}+0$

8. $2963 E+0$

8. 0434E+0

8. $2972 \mathrm{E}+0$

8. $0448 \mathrm{E}+0$

8. $2962 E+0$

8.0337E+0

8. 2964E+0

$8.0362 E+0$ 
$3.8033 \mathrm{E}+3 \quad 9.7076 \mathrm{E}-4 \quad 1.3611 \mathrm{E}+1 \quad 1.4904 \mathrm{E}-2$

$8.2581 \mathrm{E}+0 \quad 8.0135 \mathrm{E}+0$

$8.2612 \mathrm{E}+0$

$8.2554 \mathrm{E}+0$

$3.8073 \mathrm{E}+3 \quad 9.7248 \mathrm{E}-4 \quad 1.3605 \mathrm{E}+1 \quad 1.4944 \mathrm{E}-2$

$8.2528 \mathrm{E}+0 \quad 8.0140 \mathrm{E}+0$

$8.2624 \mathrm{E}+0$

$8.2682 \mathrm{E}+0$

$3.8239 \mathrm{E}+3 \quad 9.6967 \mathrm{E}-4$

1. $3605 \mathrm{E}+1$

$1.4943 \mathrm{E}-2$

8. $2577 \mathrm{E}+0$

8. $0139 \mathrm{E}+0$

$8.2622 \mathrm{E}+0$

$8.2671 \mathrm{E}+0$

$3.8364 \mathrm{E}+3 \quad 9.6934 \mathrm{E}-4$

8. $2535 \mathrm{E}+0$

8. $0139 \mathrm{E}+0$

1. $3600 \mathrm{E}+1$

1. 4951E-2

8. $2623 \mathrm{E}+0$

8. 2669E+0

$3.8397 \mathrm{E}+3 \quad 9.7362 \mathrm{E}-4$

8. $2623 \mathrm{E}+0$

8. $0137 \mathrm{E}+0$

1. $3610 \mathrm{E}+1$

8. $2619 \mathrm{E}+0$

1. $4915 \mathrm{E}-2$

8. $2436 \mathrm{E}+0$

$3.8175 \mathrm{E}+3$

8. $2592 \mathrm{E}+0$

9.7183E-4

8. $0138 \mathrm{E}+0$

1. $3636 \mathrm{E}+1$

8. $2617 \mathrm{E}+0$

1. $4913 \mathrm{E}-2$

3. $8120 \mathrm{E}+3$

9.7217E-4

1. $3617 \mathrm{E}+1$

8. $2604 \mathrm{E}+0$

8. $2567 \mathrm{E}+0$

8. $0138 \mathrm{E}+0$

8. $2619 \mathrm{E}+0$

1.4937E-2

$8.2608 \mathrm{E}+0$

3.8121E+3 9.7119E-4

1. $3612 \mathrm{E}+1$

1. $4920 \mathrm{E}-2$

8. $2571 \mathrm{E}+0$

8. $0136 \mathrm{E}+0$

$8.2616 \mathrm{E}+0$

$8.2600 \mathrm{E}+0$

1.3625E+1 1.4917E-2

$3.8155 \mathrm{E}+3 \quad 9.7416 \mathrm{E}-4$

8. $2561 \mathrm{E}+0$

$8.0138 \mathrm{E}+0$

8. $2617 \mathrm{E}+0$

$8.2525 \mathrm{E}+0$

$3.8174 \mathrm{E}+3$

9.7329E-4

1. $3614 \mathrm{E}+1$

1. $4926 \mathrm{E}-2$

8. $2565 \mathrm{E}+0$

8. $0138 \mathrm{E}+0$

8. $2620 \mathrm{E}+0$

$8.2582 \mathrm{E}+0$

$3.8206 \mathrm{E}+3$

8. $2569 \mathrm{E}+0$

9.7198E-4

8. $0138 \mathrm{E}+0$

1. $3612 \mathrm{E}+1$

8. $2620 \mathrm{E}+0$

1. 4931E-2

8. $2608 \mathrm{E}+0$

$3.8207 E+3$

$9.7302 \mathrm{E}-4$

1. $3612 \mathrm{E}+1$

1. 4920E-2

8. $2580 \mathrm{E}+0$

8. $0138 \mathrm{E}+0$

8. $2620 \mathrm{E}+0$

$8.2556 \mathrm{E}+0$

$3.8190 \mathrm{E}+3 \quad 9.7376 \mathrm{E}-4$

8. $2562 \mathrm{E}+0$

$8.0138 \mathrm{E}+0$

1. $3615 \mathrm{E}+1$

1. $4916 \mathrm{E}-2$

8. $2619 \mathrm{E}+0$

$8.2552 \mathrm{E}+0$

$3.8175 \mathrm{E}+3 \quad 9.7334 \mathrm{E}-4$

8. $2561 \mathrm{E}+0$

8. $0138 \mathrm{E}+0$

1. $3617 \mathrm{E}+1$

8. $2619 \mathrm{E}+0$

1. $4920 \mathrm{E}-2$

$3.8192 \mathrm{E}+3$

8. $2563 \mathrm{E}+0$

9.7364E-4

8. $0138 \mathrm{E}+0$

1. $3611 \mathrm{E}+1$

$8.2563 \mathrm{E}+0$

8. $2614 \mathrm{E}+0$

1.3593

8. $2615 \mathrm{E}+0$

1.3828

8. 2609E+0

1.4251

8. $2615 \mathrm{E}+0$

1.4123

8. 2617 $\mathrm{E}+0$

1.4088

8. $2615 \mathrm{E}+0$

1.4054

8. $2623 \mathrm{E}+0$

1.3818

8. $2619 \mathrm{E}+0$

1.3795

8. 2624E+0

1.3727

8. 2617E+0

1.3645

$3.8214 \mathrm{E}+3$

$8.2556 \mathrm{E}+0$

$9.7352 \mathrm{E}-4$

8. $2620 \mathrm{E}+0$

1. $4921 \mathrm{E}-2$

8. $2560 \mathrm{E}+0$

8. 2617E+0

1.3579

8. $2621 \mathrm{E}+0$

1.3559

$3.8187 \mathrm{E}+3 \quad 9.7491 \mathrm{E}-4$

8. $2539 \mathrm{E}+0$

8. $0138 \mathrm{E}+0$

1. 3609E+1

1. $4924 \mathrm{E}-2$

8. $2621 \mathrm{E}+0$

8. $2560 \mathrm{E}+0$

1. $3611 \mathrm{E}+1$

$1.4919 \mathrm{E}-2$

8. $2620 \mathrm{E}+0$

8. 2527E+0

8. $2614 \mathrm{E}+0$

1.3496

$3.8217 \mathrm{E}+3 \quad 9.7411 \mathrm{E}-4$

$8.2556 \mathrm{E}+0 \quad 8.0138 \mathrm{E}+0$

1. $3605 \mathrm{E}+1$

8. $2620 \mathrm{E}+0$

1. 4916E-2

8. $2497 \mathrm{E}+0$

8. $2615 \mathrm{E}+0$

1.3448

$3.8274 \mathrm{E}+3 \quad 9.7390 \mathrm{E}-4 \quad 1.3601 \mathrm{E}+1 \quad 1.4921 \mathrm{E}-2$

8. $2558 \mathrm{E}+0$

8. $0138 \mathrm{E}+0$

8. $2622 \mathrm{E}+0$

$8.2508 \mathrm{E}+0$

8. $2613 \mathrm{E}+0$

1.3415

3. $8278 \mathrm{E}+3$

9.7389E-4

1. $3600 \mathrm{E}+1$

1. 4926E-2

8. $2536 \mathrm{E}+0$

8. $0138 \mathrm{E}+0$

8. $2622 \mathrm{E}+0$

$8.2522 \mathrm{E}+0$

8. $2611 \mathrm{E}+0$

1.3375
$8.2965 \mathrm{E}+0$

8. $2965 E+0$

8. $2963 E+0$

8. 2964E+0

8. $2965 E+0$

8. $2965 E+0$

8. $0403 E+0$

$8.0348 \mathrm{E}+0$

8. $0443 \mathrm{E}+0$

8. $0414 \mathrm{E}+0$

8. $0413 E+0$

$8.0380 \mathrm{E}+0$

$8.0375 \mathrm{E}+0$

8. 0394E+0

8. $0376 \mathrm{E}+0$

8. $0398 \mathrm{E}+0$

8. $0416 \mathrm{E}+0$

$8.2965 E+0$

8. $0403 E+0$

$8.0388 \mathrm{E}+0$

$8.2966 \mathrm{E}+0$

8. $0400 \mathrm{E}+0$

$8.2965 E+0$

8. $0402 \mathrm{E}+0$

8. 2966E+0

$8.0415 \mathrm{E}+0$

8. 2966E+0

$8.0386 \mathrm{E}+0$

8. 2966E+0

8. $0392 \mathrm{E}+0$

8. 2966E+0

$8.0395 E+0$ 


\begin{tabular}{|c|c|c|c|c|c|c|}
\hline $\begin{array}{l}3.8241 E+3 \\
2535 E+0\end{array}$ & $\begin{array}{l}9.7472 \mathrm{E}-4 \\
8.0138 \mathrm{E}+0\end{array}$ & $\begin{array}{l}1.3597 \mathrm{E}+1 \\
8.2622 \mathrm{E}+0\end{array}$ & $\begin{array}{l}1.4924 \mathrm{E}-2 \\
8.2503 \mathrm{E}+0\end{array}$ & $\begin{array}{l}8.2613 \mathrm{E}+0 \\
1.3362\end{array}$ & $8.2967 \mathrm{E}+0$ & $8.0402 \mathrm{E}+0$ \\
\hline $\begin{array}{l}3.8274 \mathrm{E}+3 \\
8.2547 \mathrm{E}+0\end{array}$ & $\begin{array}{l}9.7445 \mathrm{E}-4 \\
8.0138 \mathrm{E}+0\end{array}$ & $\begin{array}{l}1.3596 \mathrm{E}+1 \\
8.2622 \mathrm{E}+0\end{array}$ & $\begin{array}{r}1.4 \\
8.248\end{array}$ & $\begin{array}{l}8.2614 \mathrm{E}+0 \\
1.3334\end{array}$ & $2967 E+0$ & $.0381 E+0$ \\
\hline $\begin{array}{l}3.8371 E+3 \\
2544 E+0\end{array}$ & $\begin{array}{l}9.7495 \mathrm{E}-4 \\
8.0138 \mathrm{E}+0\end{array}$ & $\begin{array}{l}1.3589 \mathrm{E}+1 \\
8.2623 \mathrm{E}+0\end{array}$ & $\begin{array}{r}1.4 \\
8.241\end{array}$ & $\begin{array}{l}8.2612 \mathrm{E}+0 \\
1.3294\end{array}$ & $.2967 E+0$ & $.0389 E+0$ \\
\hline $\begin{array}{l}3.8322 E+3 \\
.2541 E+0\end{array}$ & $\begin{array}{l}9.7454 \mathrm{E}-4 \\
8.0139 \mathrm{E}+0\end{array}$ & $\begin{array}{l}1.3595 \mathrm{E}+1 \\
8.2623 \mathrm{E}+0\end{array}$ & $\begin{array}{l}23 E-2 \\
E+0\end{array}$ & $\begin{array}{l}8.2610 \mathrm{E}+0 \\
1.3293\end{array}$ & $.2967 E+0$ & $.0389 \mathrm{E}+0$ \\
\hline $\begin{array}{l}3 E+3 \\
+0\end{array}$ & $\begin{array}{l}9.7488 \mathrm{E}-4 \\
8.0138 \mathrm{E}+0\end{array}$ & $\begin{array}{l}1.3588 \mathrm{E}+1 \\
8.2624 \mathrm{E}+0\end{array}$ & $\begin{array}{l}23 E-2 \\
E+0\end{array}$ & $\begin{array}{l}8.2609 \mathrm{E}+0 \\
1.3288\end{array}$ & $.2967 E+0$ & $.0388 \mathrm{E}+0$ \\
\hline $\begin{array}{l}5 \mathrm{E}+3 \\
+0\end{array}$ & $\begin{array}{l}9.7551 \mathrm{E}-4 \\
8.0139 \mathrm{E}+0\end{array}$ & $\begin{array}{l}1.3583 \mathrm{E}+1 \\
8.2625 \mathrm{E}+0\end{array}$ & $\begin{array}{l}21 E-2 \\
E+0\end{array}$ & $\begin{array}{l}8.2608 \mathrm{E}+0 \\
1.3258\end{array}$ & $58 \mathrm{E}+0$ & $.0386 \mathrm{E}+0$ \\
\hline $\begin{array}{l}9 E+3 \\
+0\end{array}$ & $\begin{array}{l}9.7593 \mathrm{E}-4 \\
8.0139 \mathrm{E}+0\end{array}$ & $\begin{array}{l}1.3596 \mathrm{E}+1 \\
8.2624 \mathrm{E}+0\end{array}$ & $\begin{array}{l}22 \mathrm{E}-2 \\
E+0\end{array}$ & $\begin{array}{l}8.2617 \mathrm{E}+0 \\
1.3251\end{array}$ & $2967 E+0$ & $06 \mathrm{E}+0$ \\
\hline $\begin{array}{l}0 \mathrm{E}+3 \\
+0\end{array}$ & $\begin{array}{l}9.7518 \mathrm{E}-4 \\
8.0139 \mathrm{E}+0\end{array}$ & $\begin{array}{l}1.3590 \mathrm{E}+1 \\
8.2624 \mathrm{E}+0\end{array}$ & $\begin{array}{l}24 E-2 \\
E+0\end{array}$ & $\begin{array}{l}8.2612 \mathrm{E}+0 \\
1.3242\end{array}$ & $E+0$ & $6 \mathrm{E}+0$ \\
\hline $\begin{array}{l}3.8463 \mathrm{E}+3 \\
.2531 \mathrm{E}+0\end{array}$ & $\begin{array}{l}9.7527 \mathrm{E}-4 \\
8.0139 \mathrm{E}+0\end{array}$ & $\begin{array}{l}1.3580 \mathrm{E}+1 \\
8.2626 \mathrm{E}+0\end{array}$ & $\begin{array}{l}9 \mathrm{E}-2 \\
+0\end{array}$ & $\begin{array}{l}8.2611 \mathrm{E}+0 \\
1.3229\end{array}$ & +0 & $3 \mathrm{E}+0$ \\
\hline $\begin{array}{l}3.8381 E+3 \\
.2545 E+0\end{array}$ & $\begin{array}{l}9.7563 \mathrm{E}-4 \\
8.0139 \mathrm{E}+0\end{array}$ & $\begin{array}{l}1.3589 \mathrm{E}+1 \\
8.2624 \mathrm{E}+0\end{array}$ & $\begin{array}{l}1.4918 \mathrm{E}-2 \\
8.2402 \mathrm{E}+0\end{array}$ & $\begin{array}{l}8.2615 \mathrm{E}+0 \\
1.3208\end{array}$ & $2967 E+0$ & $2 \mathrm{E}+0$ \\
\hline $\begin{array}{l}3.8399 E+3 \\
.2528 E+0\end{array}$ & $\begin{array}{l}9.7567 E-4 \\
8.0139 E+0\end{array}$ & $\begin{array}{l}1.3586 \mathrm{E}+1 \\
8.2626 \mathrm{E}+0\end{array}$ & $\begin{array}{l}1.4927 \mathrm{E}-2 \\
8.2423 \mathrm{E}+0\end{array}$ & $\begin{array}{l}8.2609 \mathrm{E}+0 \\
1.3193\end{array}$ & $7 E+0$ & $2 \mathrm{E}+0$ \\
\hline $\begin{array}{l}3.8495 E+3 \\
.2545 E+0\end{array}$ & $\begin{array}{l}9.7651 \mathrm{E}-4 \\
8.0139 \mathrm{E}+0\end{array}$ & $\begin{array}{l}1.3584 \mathrm{E}+1 \\
8.2627 \mathrm{E}+0\end{array}$ & $\begin{array}{l}1.4922 \mathrm{E}-2 \\
8.2344 \mathrm{E}+0\end{array}$ & $\begin{array}{l}8.2614 \mathrm{E}+0 \\
1.3157\end{array}$ & $7 E+0$ & $.0395 E+0$ \\
\hline $\begin{array}{l}\quad 3.8410 \mathrm{E}+3 \\
8.2531 \mathrm{E}+0\end{array}$ & $\begin{array}{l}9.7702 \mathrm{E}-4 \\
8.0139 \mathrm{E}+0\end{array}$ & $\begin{array}{l}1.3584 \mathrm{E}+1 \\
8.2627 \mathrm{E}+0\end{array}$ & $\begin{array}{l}1.4924 \mathrm{E}-2 \\
8.2383 \mathrm{E}+0\end{array}$ & $\begin{array}{l}8.2620 \mathrm{E}+0 \\
1.3122\end{array}$ & . 2967E+0 & $.0407 E+0$ \\
\hline $\begin{array}{l}3.8494 \mathrm{E}+3 \\
8.2524 \mathrm{E}+0\end{array}$ & $\begin{array}{l}9.7688 \mathrm{E}-4 \\
8.0140 \mathrm{E}+0\end{array}$ & $\begin{array}{l}1.3579 \mathrm{E}+1 \\
8.2628 \mathrm{E}+0\end{array}$ & $\begin{array}{l}1.4926 \mathrm{E}-2 \\
8.2358 \mathrm{E}+0\end{array}$ & $\begin{array}{l}8.2619 \mathrm{E}+0 \\
1.3027\end{array}$ & . 2967E+0 & $.0392 E+0$ \\
\hline $\begin{array}{l}3.8500 \mathrm{E}+3 \\
8.2518 \mathrm{E}+0\end{array}$ & $\begin{array}{l}9.7771 \mathrm{E}-4 \\
8.0140 \mathrm{E}+0\end{array}$ & $\begin{array}{l}1.3582 \mathrm{E}+1 \\
8.2630 \mathrm{E}+0\end{array}$ & $\begin{array}{l}1.4933 \mathrm{E}-2 \\
8.2367 \mathrm{E}+0\end{array}$ & $\begin{array}{l}8.2625 \mathrm{E}+0 \\
1.3024\end{array}$ & $.2966 \mathrm{E}+0$ & $3.0420 \mathrm{E}+0$ \\
\hline $\begin{array}{l}3.8647 E+3 \\
8.2529 E+0\end{array}$ & $\begin{array}{l}9.7887 \mathrm{E}-4 \\
8.0141 \mathrm{E}+0\end{array}$ & $\begin{array}{l}1.3573 \mathrm{E}+1 \\
8.2633 \mathrm{E}+0\end{array}$ & $\begin{array}{l}1.4934 \mathrm{E}-2 \\
8.2280 \mathrm{E}+0\end{array}$ & $\begin{array}{l}8.2632 \mathrm{E}+0 \\
1.2965\end{array}$ & $8.2966 \mathrm{E}+0$ & $8.0409 E+0$ \\
\hline $\begin{array}{l}\quad 3.8643 E+3 \\
8.2509 E+0\end{array}$ & $\begin{array}{l}9.8074 \mathrm{E}-4 \\
8.0141 \mathrm{E}+0\end{array}$ & $\begin{array}{l}1.3576 \mathrm{E}+1 \\
8.2632 \mathrm{E}+0\end{array}$ & $\begin{array}{l}1.4921 \mathrm{E}-2 \\
8.2234 \mathrm{E}+0\end{array}$ & $\begin{array}{l}8.2627 \mathrm{E}+0 \\
1.2885\end{array}$ & $8.2966 \mathrm{E}+0$ & $8.0423 E+0$ \\
\hline $\begin{aligned} & 3.8725 E+3 \\
8 . & 2509 E+0\end{aligned}$ & $\begin{array}{l}9.8016 \mathrm{E}-4 \\
\text { 8. } 0142 \mathrm{E}+0\end{array}$ & $\begin{array}{l}1.3573 \mathrm{E}+1 \\
8.2636 \mathrm{E}+0\end{array}$ & $\begin{array}{l}1.4935 \mathrm{E}-2 \\
8.2237 \mathrm{E}+0\end{array}$ & $\begin{array}{l}8.2635 \mathrm{E}+0 \\
1.2802\end{array}$ & $8.2966 \mathrm{E}+0$ & $8.0409 E+0$ \\
\hline $\begin{array}{l}3.8894 \mathrm{E}+3 \\
\text { 8. } 2491 \mathrm{E}+0\end{array}$ & $\begin{array}{l}9.8301 \mathrm{E}-4 \\
8.0143 \mathrm{E}+0\end{array}$ & $\begin{array}{l}1.3556 \mathrm{E}+1 \\
8.2641 \mathrm{E}+0\end{array}$ & $\begin{array}{l}38 \mathrm{E}-2 \\
=+0\end{array}$ & $3 E+0$ & $5 \mathrm{E}+0$ & $.0425 \mathrm{E}+0$ \\
\hline
\end{tabular}


$3.8811 \mathrm{E}+3 \quad 9.8345 \mathrm{E}-4 \quad 1.3566 \mathrm{E}+1 \quad 1.4936 \mathrm{E}-2$ $8.2483 \mathrm{E}+0 \quad 8.0143 \mathrm{E}+0$

$3.8884 \mathrm{E}+3$

8. $2449 \mathrm{E}+0$

$9.8608 \mathrm{E}-4$

8. $2641 \mathrm{E}+0$

8. $2194 \mathrm{E}+0$

1. $3557 \mathrm{E}+1$

1. $4928 \mathrm{E}-2$

8. $0144 \mathrm{E}+0$

8. $2643 \mathrm{E}+0$

8. $2066 \mathrm{E}+0$

$3.9083 E+3$

9. $8848 \mathrm{E}-4$

1. $3544 \mathrm{E}+1$

8. $0145 \mathrm{E}+0$

8. $2650 \mathrm{E}+0$

1. $4940 \mathrm{E}-2$

8. $2439 \mathrm{E}+0$

$9.8848 \mathrm{E}-4$

$3.8973 \mathrm{E}+3$

8. $0145 \mathrm{E}+0$

1. $3547 \mathrm{E}+1$

8. $1984 \mathrm{E}+0$

8. $2433 \mathrm{E}+0$

9. $9027 \mathrm{E}-4$

$3.9061 \mathrm{E}+3$

8. $2421 \mathrm{E}+0$

8. $0146 \mathrm{E}+0$

1. $3551 \mathrm{E}+1$

8. $2650 \mathrm{E}+0$

$1.4934 \mathrm{E}-2$

8. $2648 \mathrm{E}+0$

$8.1984 \mathrm{E}+0$

1. $4935 \mathrm{E}-2$

$3.9066 \mathrm{E}+3$

8. $2407 \mathrm{E}+0$

9. $9010 \mathrm{E}-4$

1. $3544 \mathrm{E}+1$

$8.1952 \mathrm{E}+0$

8. $0146 \mathrm{E}+0$

8. $2651 \mathrm{E}+0$

1. $4941 \mathrm{E}-2$

8. $1963 \mathrm{E}+0$

$3.9053 \mathrm{E}+3 \quad 9.8988 \mathrm{E}-4$

1. $3551 \mathrm{E}+1$

1. 4935E-2

$8.2417 \mathrm{E}+0$

8. $0146 \mathrm{E}+0$

8. $2650 \mathrm{E}+0$

8. $1971 \mathrm{E}+0$

$3.9089 \mathrm{E}+3 \quad 9.9155 \mathrm{E}-4$

1. $3547 \mathrm{E}+1$

1. 4941E-2

8. $2401 \mathrm{E}+0$

8. $0147 \mathrm{E}+0$

$8.2653 \mathrm{E}+0$

$8.1935 \mathrm{E}+0$

1. $3548 \mathrm{E}+1$

$3.9021 \mathrm{E}+3 \quad 9.8980 \mathrm{E}-4$

8. $2417 \mathrm{E}+0$

8. $0146 \mathrm{E}+0$

8. $2650 \mathrm{E}+0$

1. 4940E-2

$8.1972 \mathrm{E}+0$

$3.9059 \mathrm{E}+3$

9. $9015 \mathrm{E}-4$

1. $3548 \mathrm{E}+1$

8. $2415 \mathrm{E}+0$

8. $0146 \mathrm{E}+0$

8. $2651 \mathrm{E}+0$

1. $4939 \mathrm{E}-2$

8. $1957 \mathrm{E}+0$

$3.9012 \mathrm{E}+3 \quad 9.8975 \mathrm{E}-4$

1. $3551 \mathrm{E}+1$

1.4937E-2

8. $2418 \mathrm{E}+0$

8. $0146 \mathrm{E}+0$

8. $2650 \mathrm{E}+0$

8. $1980 \mathrm{E}+0$

$3.9026 \mathrm{E}+3$

9. $9010 \mathrm{E}-4$

1. 3551E+1

1. 4937E-2

8. $2414 \mathrm{E}+0$

8. $0146 \mathrm{E}+0$

8. $2650 \mathrm{E}+0$

8. $1967 \mathrm{E}+0$

1. $3548 \mathrm{E}+1$

$3.9047 \mathrm{E}+3$

9. $9013 \mathrm{E}-4$

8. $2413 \mathrm{E}+0$

8. $0146 \mathrm{E}+0$

$8.2651 \mathrm{E}+0$

1. $4939 \mathrm{E}-2$

$8.1963 \mathrm{E}+0$

$3.9046 \mathrm{E}+3 \quad 9.9057 \mathrm{E}-4$

8. $2411 \mathrm{E}+0$

8. $0146 \mathrm{E}+0$

1. 3547E+1

1. $4938 \mathrm{E}-2$

8. $2651 \mathrm{E}+0$

8. $1950 \mathrm{E}+0$

8. $2662 \mathrm{E}+0$

1.2425

$3.9063 \mathrm{E}+3 \quad 9.9084 \mathrm{E}-4$

1. $3548 \mathrm{E}+1$

1. $4939 \mathrm{E}-2$

8. $2408 \mathrm{E}+0$

8. $0146 \mathrm{E}+0$

8. $2652 \mathrm{E}+0$

$8.1949 \mathrm{E}+0$

8. 2664E+0

1.2423

$3.9037 \mathrm{E}+3 \quad 9.9014 \mathrm{E}-4$

1. 3549E+1

1. 4939E-2

8. $2413 \mathrm{E}+0$

8. $0146 \mathrm{E}+0$

8. $2651 \mathrm{E}+0$

8. $1971 \mathrm{E}+0$

8. 2662 $\mathrm{E}+0$

1.2423

$3.9048 \mathrm{E}+3 \quad 9.9053 \mathrm{E}-4$

8. $2409 \mathrm{E}+0$

8. $0146 \mathrm{E}+0$

1. $3548 \mathrm{E}+1$

$1.4939 \mathrm{E}-2$

8. $2651 \mathrm{E}+0$

$8.1954 \mathrm{E}+0$

8. 2663E+0

1.2422

$3.9043 \mathrm{E}+3 \quad 9.9031 \mathrm{E}-4$

8. $2412 \mathrm{E}+0 \quad 8.0146 \mathrm{E}+0$

1. $3549 \mathrm{E}+1$

1. $4939 \mathrm{E}-2$

8. $2651 \mathrm{E}+0$

$8.1964 \mathrm{E}+0$

8. $2663 \mathrm{E}+0$

1.2422

3. $9033 \mathrm{E}+3$

8. $2413 \mathrm{E}+0$

9. $9022 \mathrm{E}-4$

1. $3548 \mathrm{E}+1$

8. $0146 \mathrm{E}+0$

8. $2651 \mathrm{E}+0$

$1.4939 \mathrm{E}-2$

8. $1963 \mathrm{E}+0$

$8.2662 \mathrm{E}+0$

1.2421

8. $2965 \mathrm{E}+0$

8. $0433 \mathrm{E}+0$

1.2694

8. $2653 \mathrm{E}+0$

$8.2965 \mathrm{E}+0$

8. $0441 \mathrm{E}+0$

$8.2964 \mathrm{E}+0$

8. $0471 \mathrm{E}+0$

$8.2965 \mathrm{E}+0$

8. $0469 \mathrm{E}+0$

$8.2964 \mathrm{E}+0$

8. $0463 \mathrm{E}+0$

$8.2964 \mathrm{E}+0$

8. $0474 \mathrm{E}+0$

$8.2964 \mathrm{E}+0$

8. $0466 \mathrm{E}+0$

8. 2964E+0

8. $0466 \mathrm{E}+0$

$8.2964 \mathrm{E}+0$

8. $0466 \mathrm{E}+0$

$8.2964 \mathrm{E}+0$

8. $0466 \mathrm{E}+0$

$8.2964 \mathrm{E}+0$

8. 0467E+0

$8.2964 \mathrm{E}+0$

$8.0468 \mathrm{E}+0$

8. 2964E+0

8. $0470 \mathrm{E}+0$

8. 2964E+0

$8.0468 \mathrm{E}+0$

sum squares $=1.7932 \mathrm{E}-4$ 
Minmax constraint was active.

OPAR $(1 e+8)$

NEWTON (plot)

$\mathrm{k} 1$

$\mathrm{k} 2$

k8

$\mathrm{k} 9$

$k 3$

$\mathrm{k} 4$

$k 11$

k5

R.M.S. [\%]

k6

k7

Initial values:--.------

3.9033E+3 9.9022E-4 $1.3548 \mathrm{E}+1 \quad 1.4939 \mathrm{E}-2$

$8.2413 \mathrm{E}+0 \quad 8.0146 \mathrm{E}+0 \quad 8.2651 \mathrm{E}+0 \quad 8.1963 \mathrm{E}+0$

$8.2662 \mathrm{E}+0$

$8.2964 \mathrm{E}+0$

$8.0465 \mathrm{E}+0$

$\pm 0.0000 \mathrm{E}+0 \quad \pm 0.0000 \mathrm{E}+0 \quad \pm 0.0000 \mathrm{E}+0 \quad \pm 0.0000 \mathrm{E}+0$

1.2421

$\pm 0.0000 \mathrm{E}+0 \quad \pm 0.0000 \mathrm{E}+0 \quad \pm 0.0000 \mathrm{E}+0 \quad \pm 0.0000 \mathrm{E}+0$

$\pm 0.0000 \mathrm{E}+0 \quad \pm 0.0000 \mathrm{E}+0 \quad \pm 0.0000 \mathrm{E}+0$

$3.9033 \mathrm{E}+3 \quad 9.9130 \mathrm{E}-4 \quad 1.3548 \mathrm{E}+1 \quad 1.4939 \mathrm{E}-2$

$\begin{array}{llll}8.2428 \mathrm{E}+0 & 8.0146 \mathrm{E}+0 & 8.2650 \mathrm{E}+0 & 8.2019 \mathrm{E}+0\end{array}$

$8.2712 \mathrm{E}+0$

$8.2963 E+0$

$8.0361 \mathrm{E}+0$

$\pm 3.4145 \mathrm{E}+0 \pm 4.2980 \mathrm{E}-5 \quad \pm 1.7804 \mathrm{E}-2 \quad \pm 6.4758 \mathrm{E}-5$

1.2412

$\pm 4.8418 \mathrm{E}-2 \pm 8.0427 \mathrm{E}-4 \pm 5.5243 \mathrm{E}-3 \pm 1.8867 \mathrm{E}-1$
$3.9033 \mathrm{E}+3$
$9.9033 \mathrm{E}-4$
1. $3548 \mathrm{E}+1$
$1.4939 \mathrm{E}-2$
$8.2684 \mathrm{E}+0$
$8.2964 \mathrm{E}+0$
$8.0365 \mathrm{E}+0$

$8.2457 \mathrm{E}+0$

$8.0146 \mathrm{E}+0 \quad 8.2650 \mathrm{E}+0 \quad 8.1992 \mathrm{E}+0$

1.2408

$\pm 1.6768 \mathrm{E}+0 \quad \pm 1.1218 \mathrm{E}-4 \quad \pm 1.0896 \mathrm{E}-2 \quad \pm 4.1417 \mathrm{E}-5$

$\pm 3.4214 \mathrm{E}-1 \quad \pm 8.8846 \mathrm{E}-3 \quad \pm 3.3118 \mathrm{E}-1$ $\pm 3.6743 \mathrm{E}-1 \pm 5.7653 \mathrm{E}-3 \pm 9.8577 \mathrm{E}-3 \pm 3.4853 \mathrm{E}-1$

$3.9033 \mathrm{E}+3 \quad 9.9071 \mathrm{E}-4 \quad 1.3548 \mathrm{E}+1 \quad 1.4935 \mathrm{E}-2$ $8.2639 \mathrm{E}+0$

$+3.4952 \mathrm{E}+0+3.3925 \mathrm{E}-6+9.5354 \mathrm{E}-3+9.2221 \mathrm{E}-5$ $\pm 3.1784 \mathrm{E}-1 \pm 2.8271 \mathrm{E}-3 \pm 4.0814 \mathrm{E}-3 \pm 9.7279 \mathrm{E}-2$

$3.9033 \mathrm{E}+3 \quad 9.9071 \mathrm{E}-4 \quad 1.3548 \mathrm{E}+1 \quad 1.4935 \mathrm{E}-2$ 8.2639E+0 8.0144E+0 8.2651E+0 8.2007E+0 $\pm 2.2144 \mathrm{E}+0 \quad \pm 2.6026 \mathrm{E}-5 \quad \pm 1.7397 \mathrm{E}-2 \quad \pm 8.1379 \mathrm{E}-5$ $\pm 1.9155 \mathrm{E}-1 \pm 1.3202 \mathrm{E}-3 \pm 4.0704 \mathrm{E}-3 \quad \pm 1.1149 \mathrm{E}-1$

sum squares $=1.7829 \mathrm{E}-4$

Covariance Matrix:

$\begin{array}{ccc}\mathrm{k} 10 \_ & -\mathrm{k} 1 \ldots & -\mathrm{k} 2 \\ \mathrm{k} 1 & 1.000 & 0.133 \\ 0.276 & 0.080 & \\ \mathrm{k} 2 & 0.133 & 1.000 \\ 0.261 & 0.486 & \\ \mathrm{k} 3 & -0.432 & -0.203 \\ 0.024 & -0.095 & \\ \mathrm{k} 4 & -1.242 & -0.142 \\ 0.259 & -0.024 & \\ \mathrm{k} 5 & 0.036 & 0.017 \\ 0.011 & 0.007 & \\ \mathrm{k} 6 & -0.031 & -0.006 \\ 0.006 & -0.002 & \\ \mathrm{k} 7 & -0.095 & -0.358 \\ 0.123 & -0.169 & \\ \mathrm{k} 8 & -0.044 & 0.093 \\ 0.022 & 0.047 & \\ \mathrm{k} 9 & -2.081 & 0.524 \\ 0.026 & -0.227 & \end{array}$$$
\text { k3 }
$$

k4
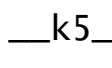

$$
-0.432
$$

$-1.242$

0.036

$-0.203-0.142$

0.017

1.000

0.316

$-0.068$

$0.316-1.000$

0.028

$-0.068$

0.028

$0.065-0.021$

$0.221-0.077$

$-1.000$

1.01

2.31

$0.076-0.026$

1.480

$0.409-1.843$

$0.060 \quad-0.041 \quad-0.409$

$8.2969 \mathrm{E}+0$

8.0393E+0 1.2385

8. $2498 \mathrm{E}+0$

$8.2969 \mathrm{E}+0$

8. $0393 \mathrm{E}+0$ 1.2385

$\pm 3.5179 \mathrm{E}-1 \quad \pm 8.8772 \mathrm{E}-3 \quad \pm 2.8821 \mathrm{E}-1$ $\pm 4.4235 \mathrm{E}-1 \pm 1.0413 \mathrm{E}-2 \quad \pm 1.2134 \mathrm{E}-1$ $0.026-0.227$ 


$\begin{array}{ccccccccccc}\mathrm{k} 10 & 0.276 & -0.261 & -0.024 & 0.259 & -0.011 & 0.006 & 0.123 & -0.022 & -0.026 \\ 1.000 & -0.709 & & & & & & & & & \\ \mathrm{k} 11 & 0.080 & 0.486 & -0.095 & -0.024 & 0.007 & -0.002 & -0.169 & 0.047 & -0.227 & - \\ 0.709 & 1.000 & & & & & & & & & \end{array}$

PLOT (spec)

PLOT (resi)

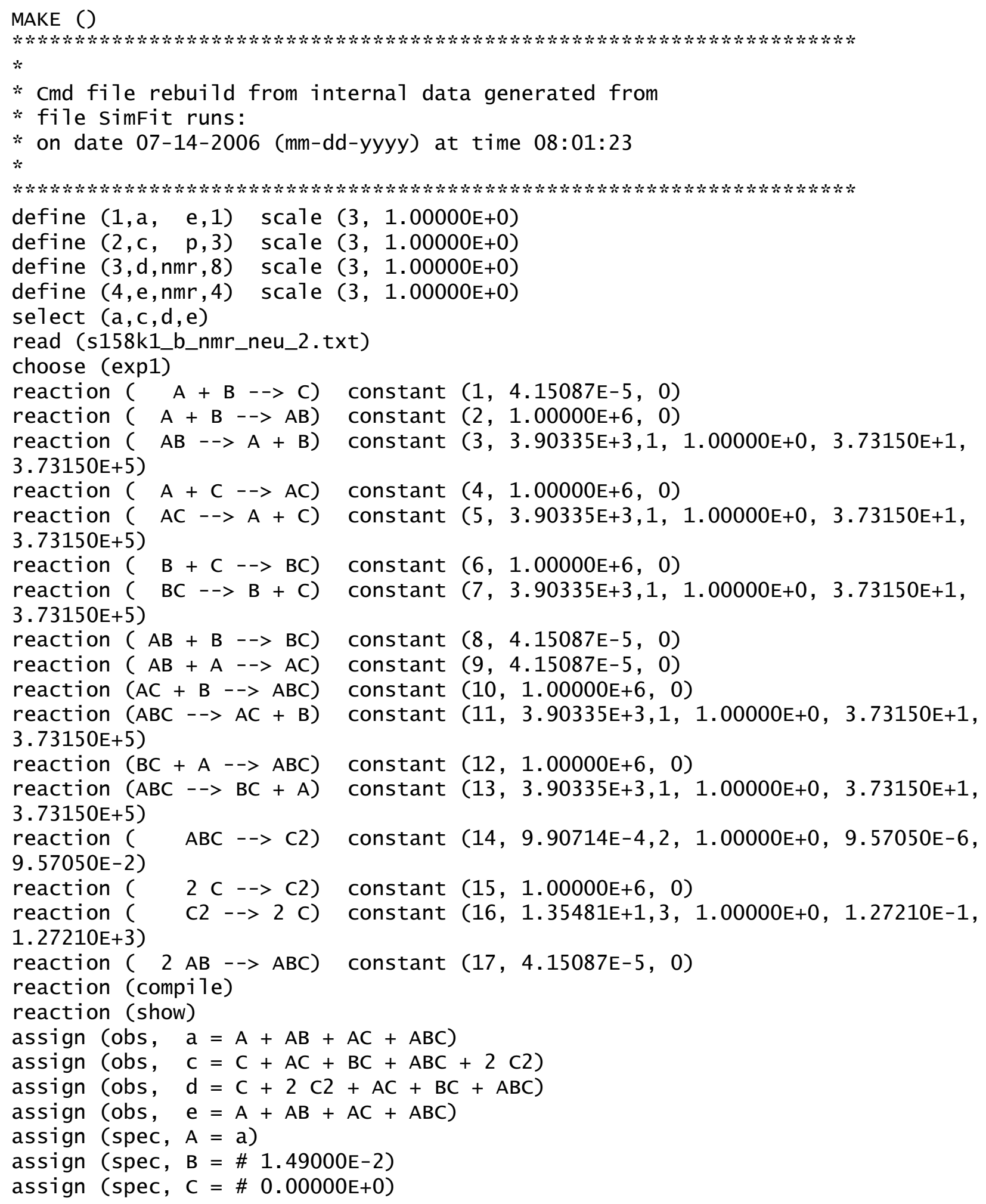


delta (d, C, 8.24976E+0, 5.00000E+0, 7.00000E+0, 9.00000E+0)

delta (d, AC, 8.24976E+0, 5.00000E+0, 7.00000E+0, 9.00000E+0)

de1ta (d, BC, 8.03930E+0, 7.00000E+0, 7.00000E+0, 9.00000E+0)

delta (d, ABC, 8.26386E+0, 8.00000E+0, 7.00000E+0, 9.00000E+0)

de1ta (d, C2, 8.29691E+0, 6.00000E+0, 7.00000E+0, 9.00000E+0)

delta (e, A, 8.01443E+0, 9.00000E+0, 7.00000E+0, 9.00000E+0)

de1ta (e, $A B, 8.26510 \mathrm{E}+0,1.00000 \mathrm{E}+1,7.00000 \mathrm{E}+0,9.00000 \mathrm{E}+0)$

delta (e, AC, 8.26510E+0, 1.00000E+1, 7.00000E+0, 9.00000E+0)

de1ta (e, ABC, 8.20066E+0, 1.10000E+1, 7.00000E+0, 9.00000E+0)

* The initial concentration of observable a in experiment 1

* of file s158k1_b_nmr_neu_2.txt was assumed to be $1.49346062475697 \mathrm{E}-2 \mathrm{~mol} / 1$ and was forced

* to be between $1.46380952380952 \mathrm{E}-2$ and $.0161385 \mathrm{~mol} / 1$.

Czero (4,a,1,1, 1.46381E-2, 1.61385E-2, 1.49346E-2)

time $(h)$

conc (mM)

win $(0,70,10,0,-1,16,2,0)$

nmrwin $(0,70,10,0,8.05,8.3, .1,0)$

$\operatorname{dim}(11)$

integ (stiff, .0000001, 32, .05, 500, 1500)

plot

\section{Excel macro for transformation of NMR-data into SimFit data files}

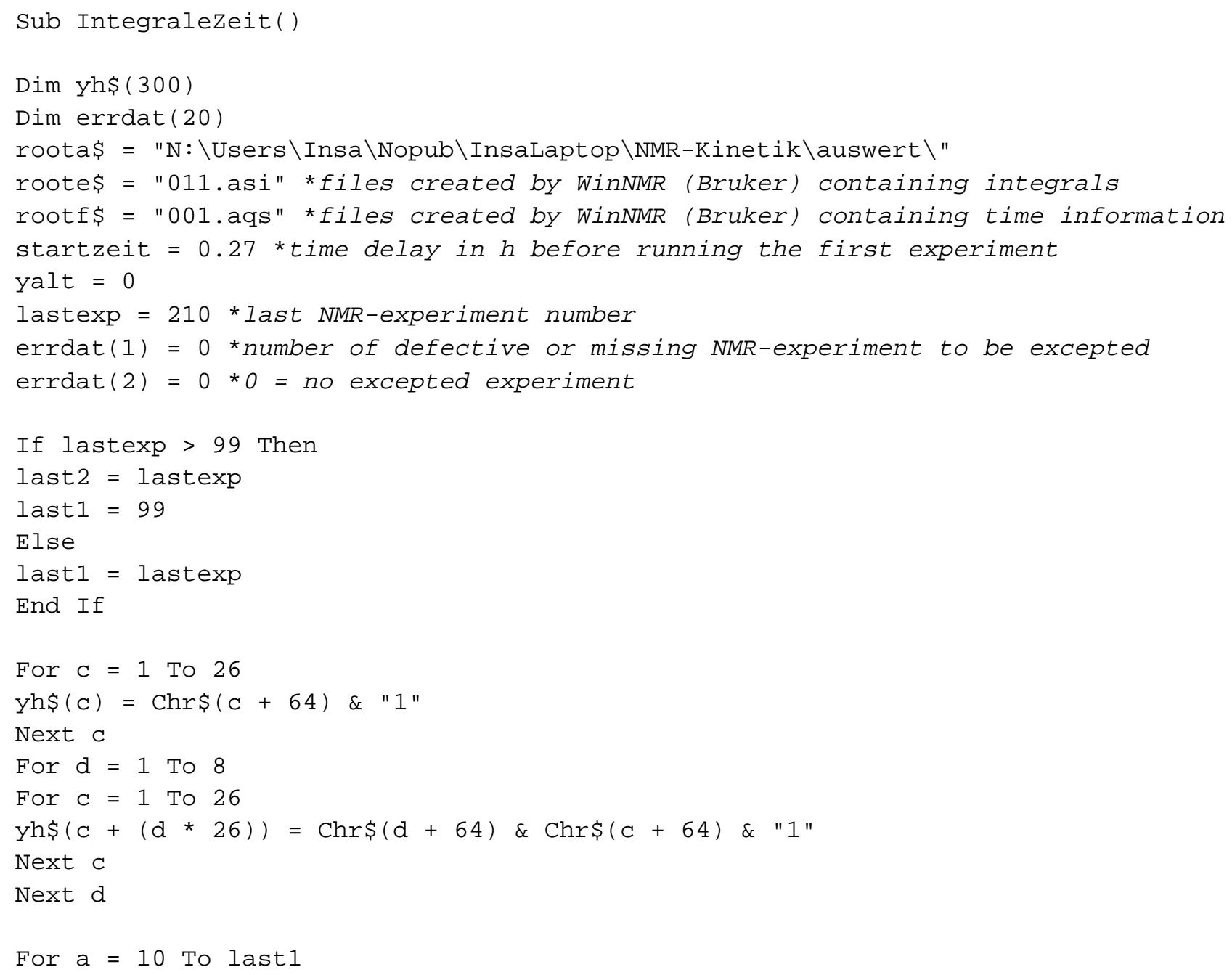




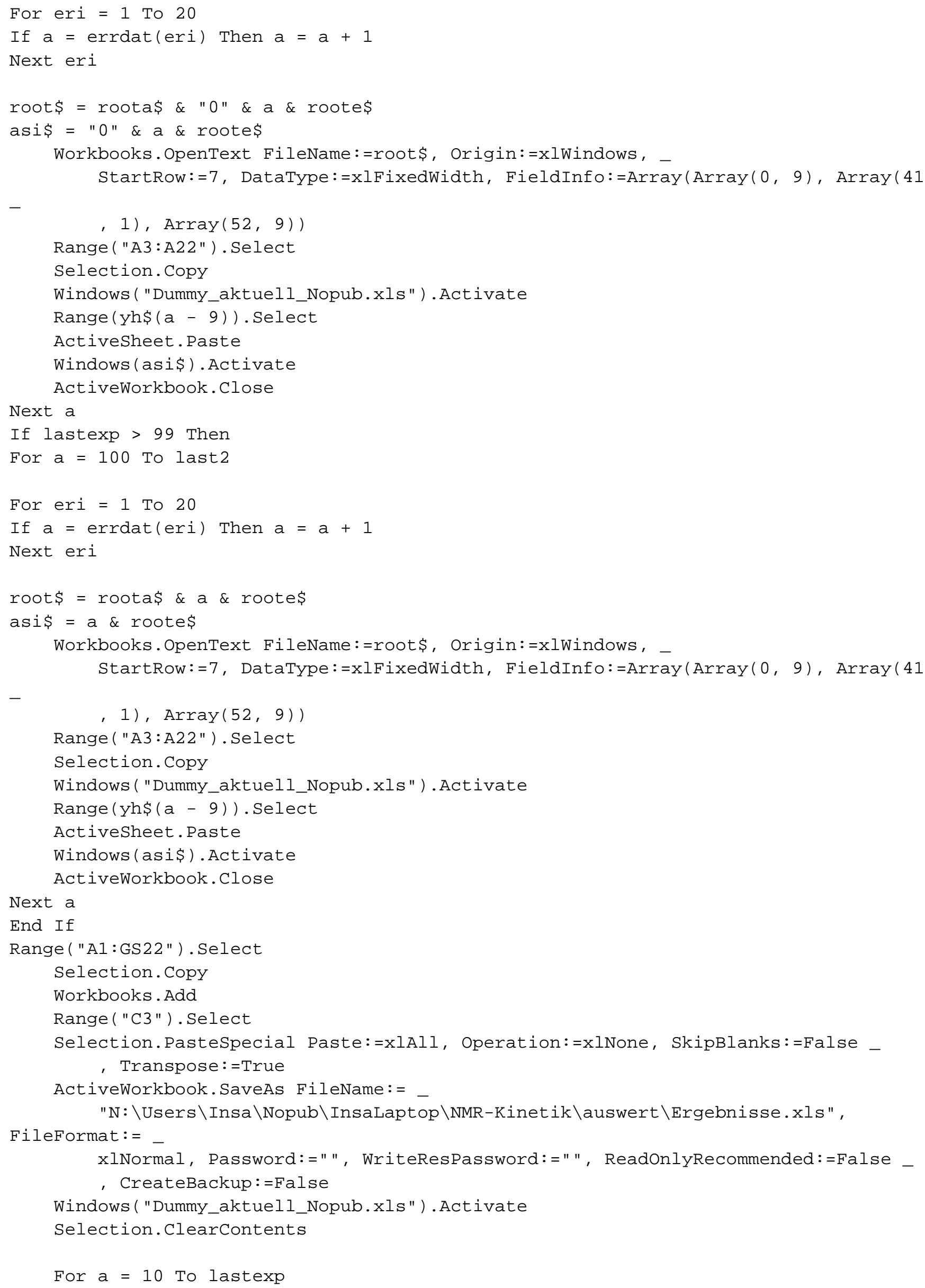




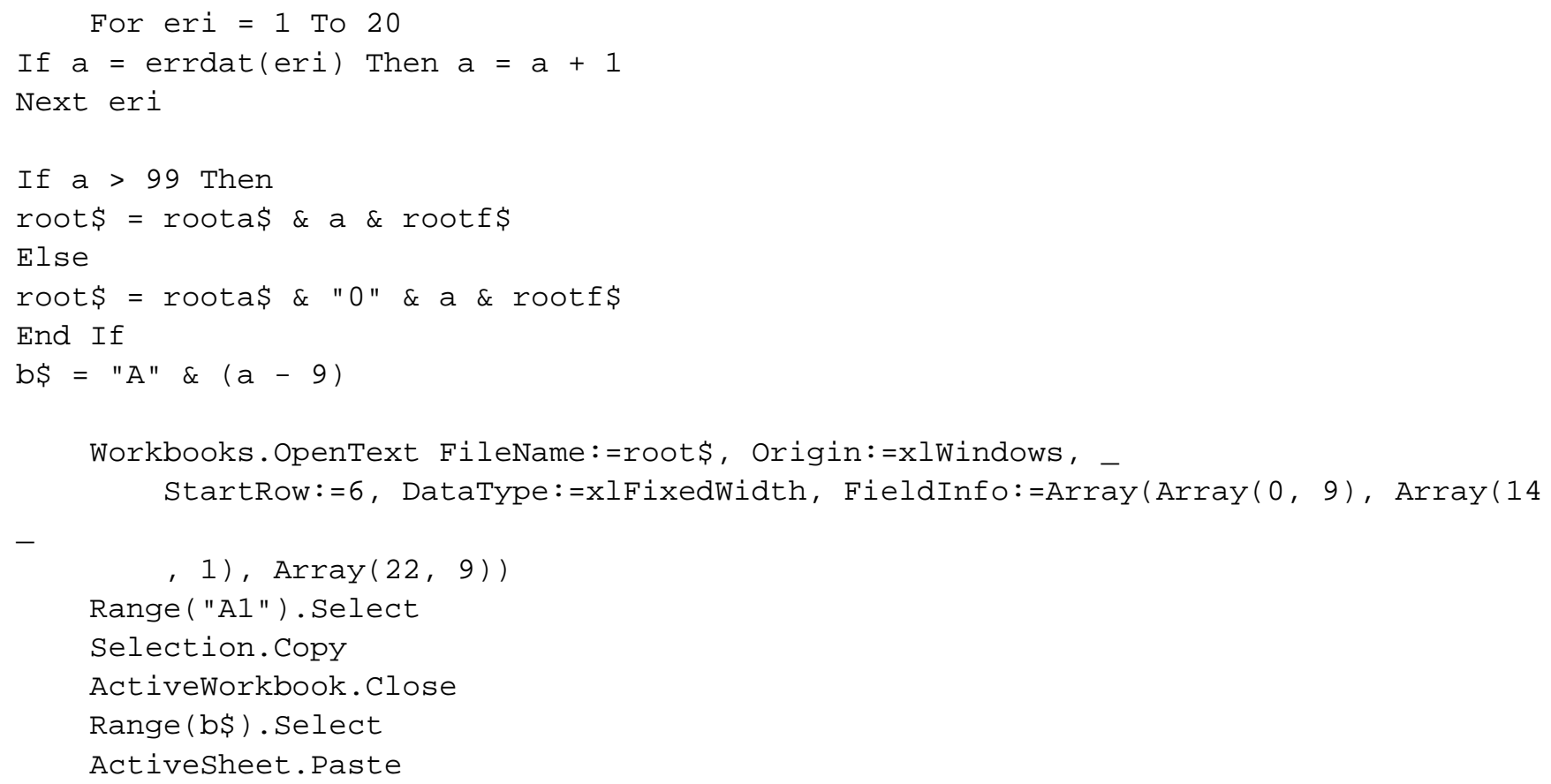

\section{End Sub}


Concentration-time and chemical shift-time profiles for the reaction of 1 with $2 a$

( $\mathrm{c}=15 \mathrm{mM}$ for both precursors, $\mathrm{C}_{2} \mathrm{D}_{2} \mathrm{Cl}_{4}$; continuous lines represent theoretical curves) $\mathbf{T}=323 \mathrm{~K}$
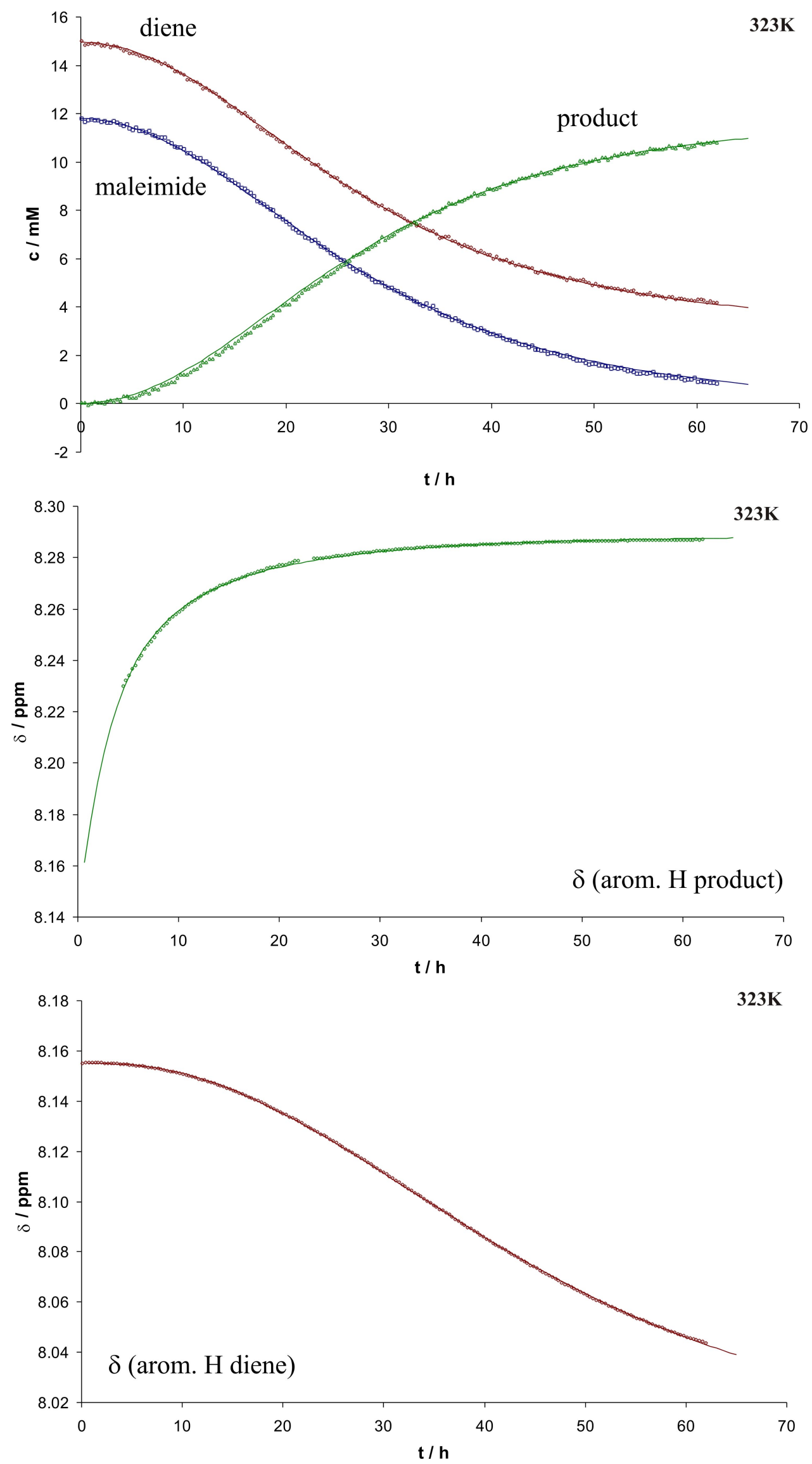
$\mathbf{T}=343 \mathrm{~K}$
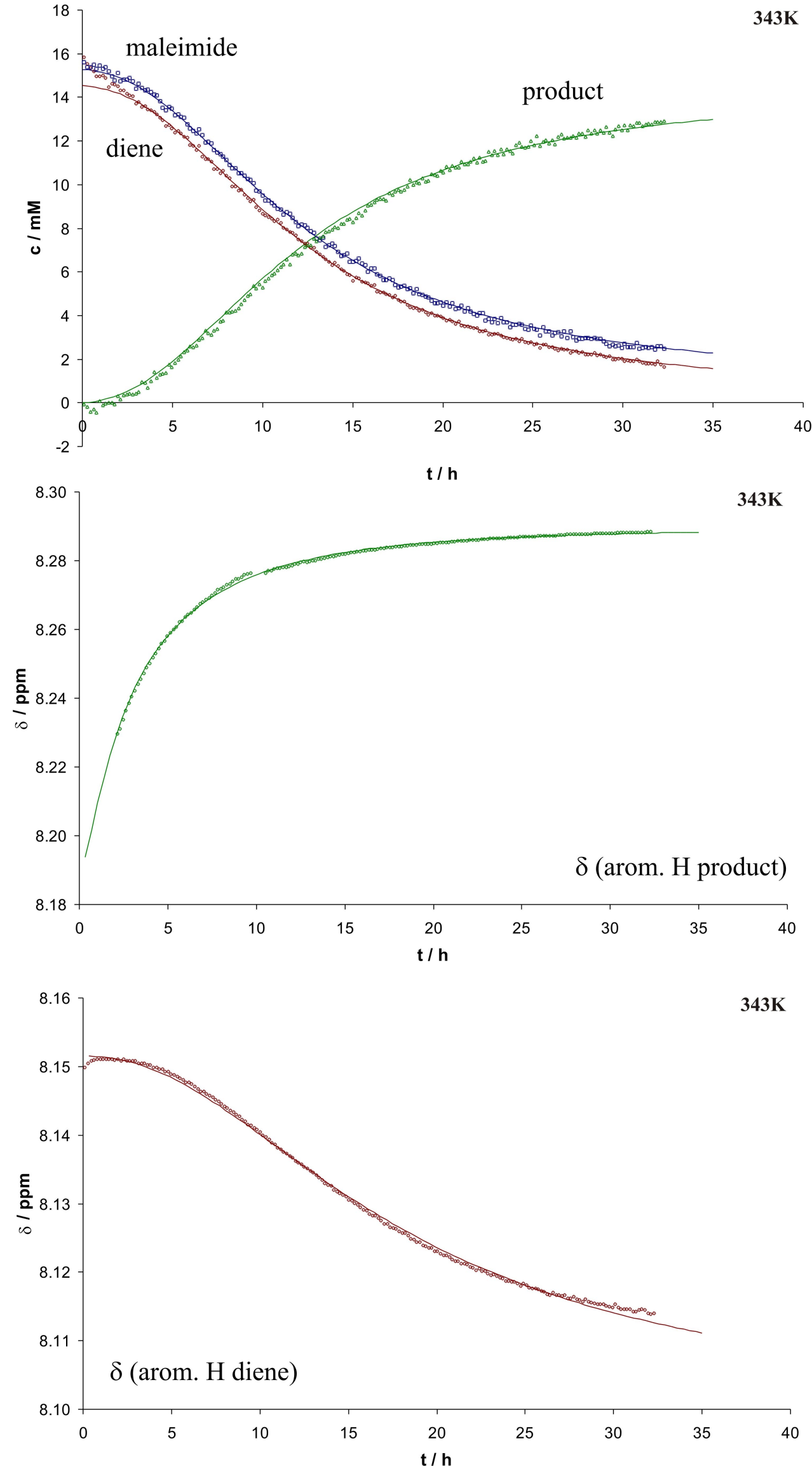
Concentration-time and chemical shift-time profiles for the reaction of 1 with $2 b$

( $c=15 \mathrm{mM}$ for both precursors, $\mathrm{C}_{2} \mathrm{D}_{2} \mathrm{Cl}_{4}$; continuous lines represent theoretical curves)

$\mathrm{T}=303 \mathrm{~K}$
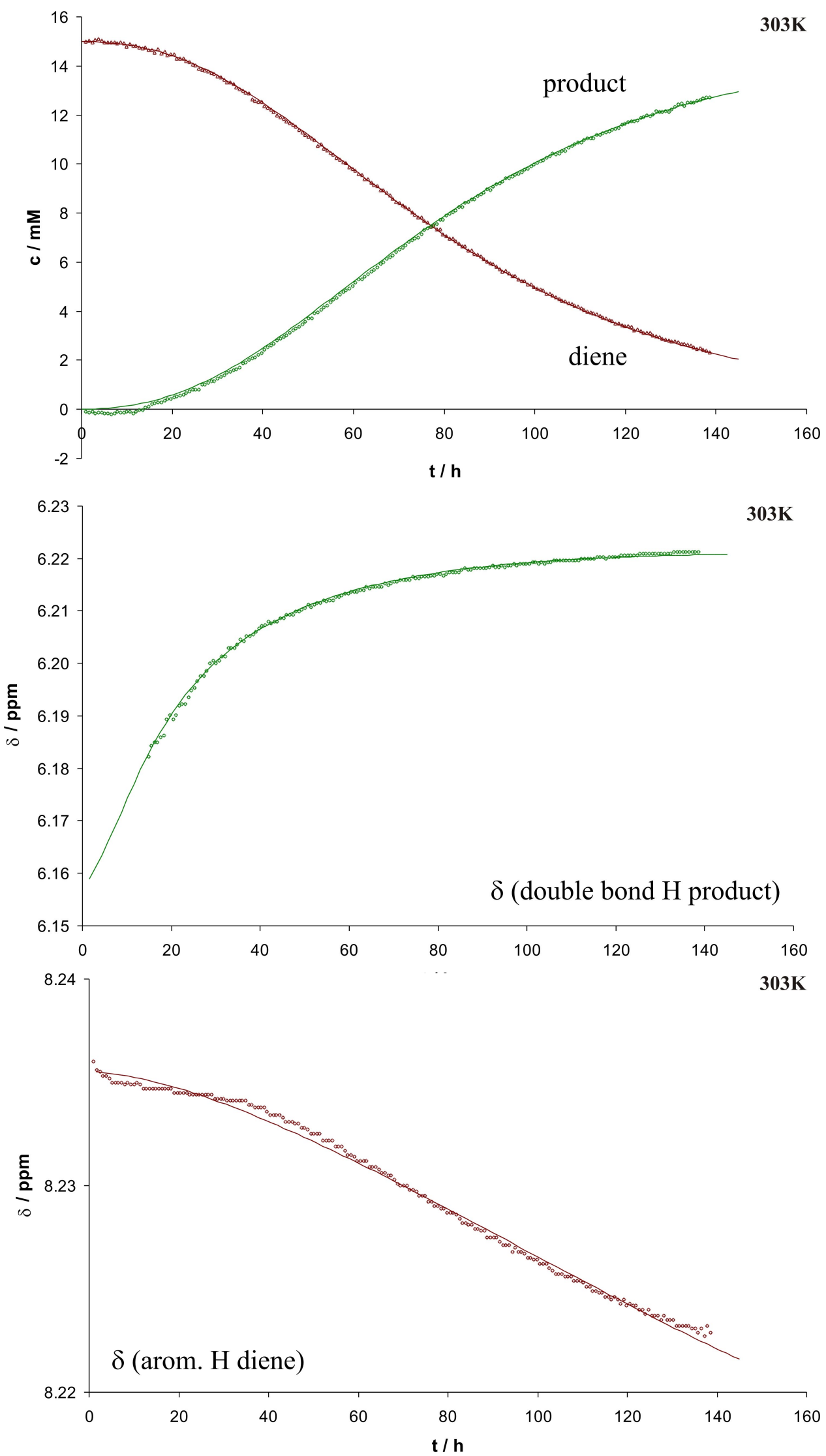

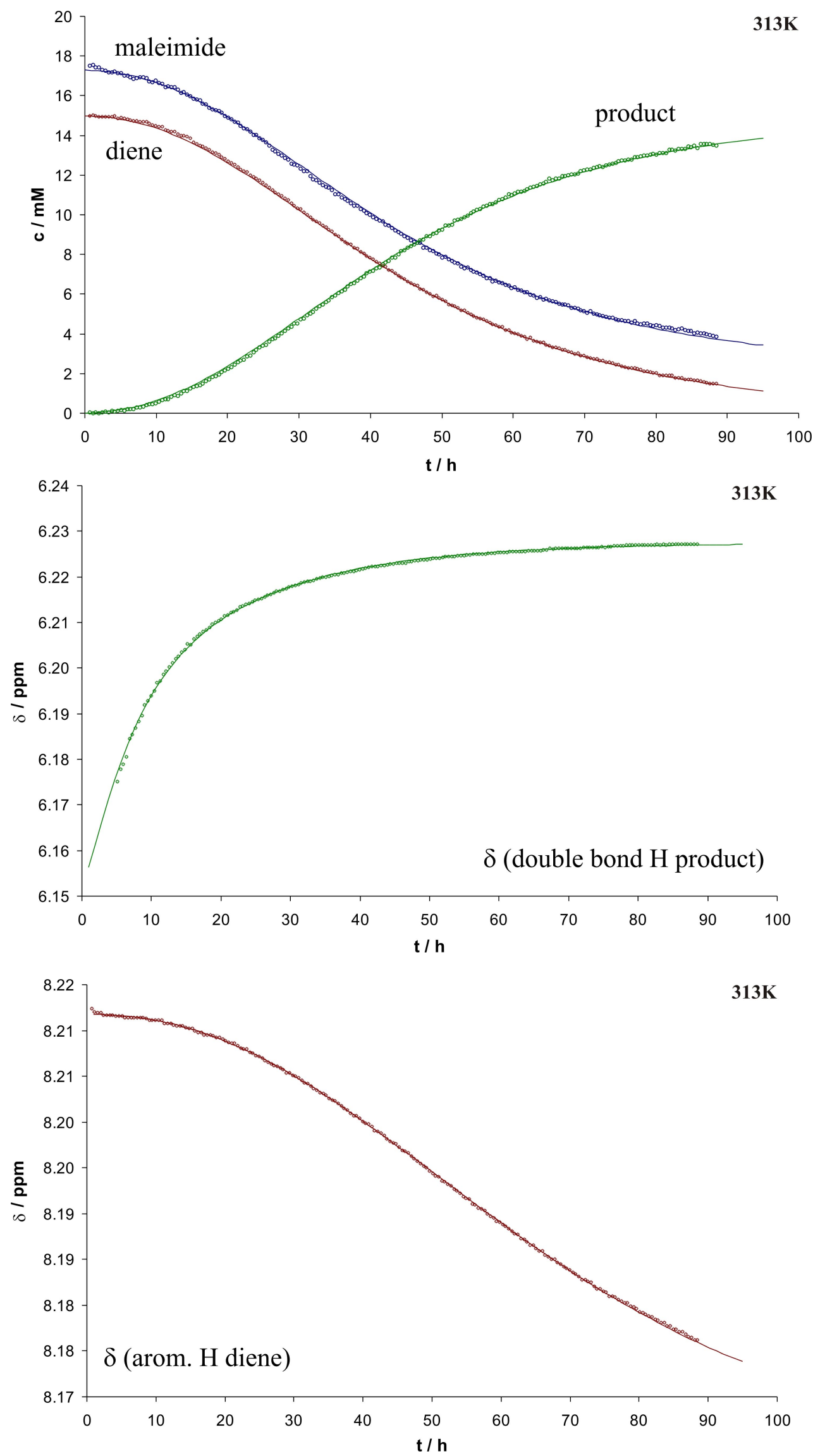
$\mathrm{T}=318 \mathrm{~K}$
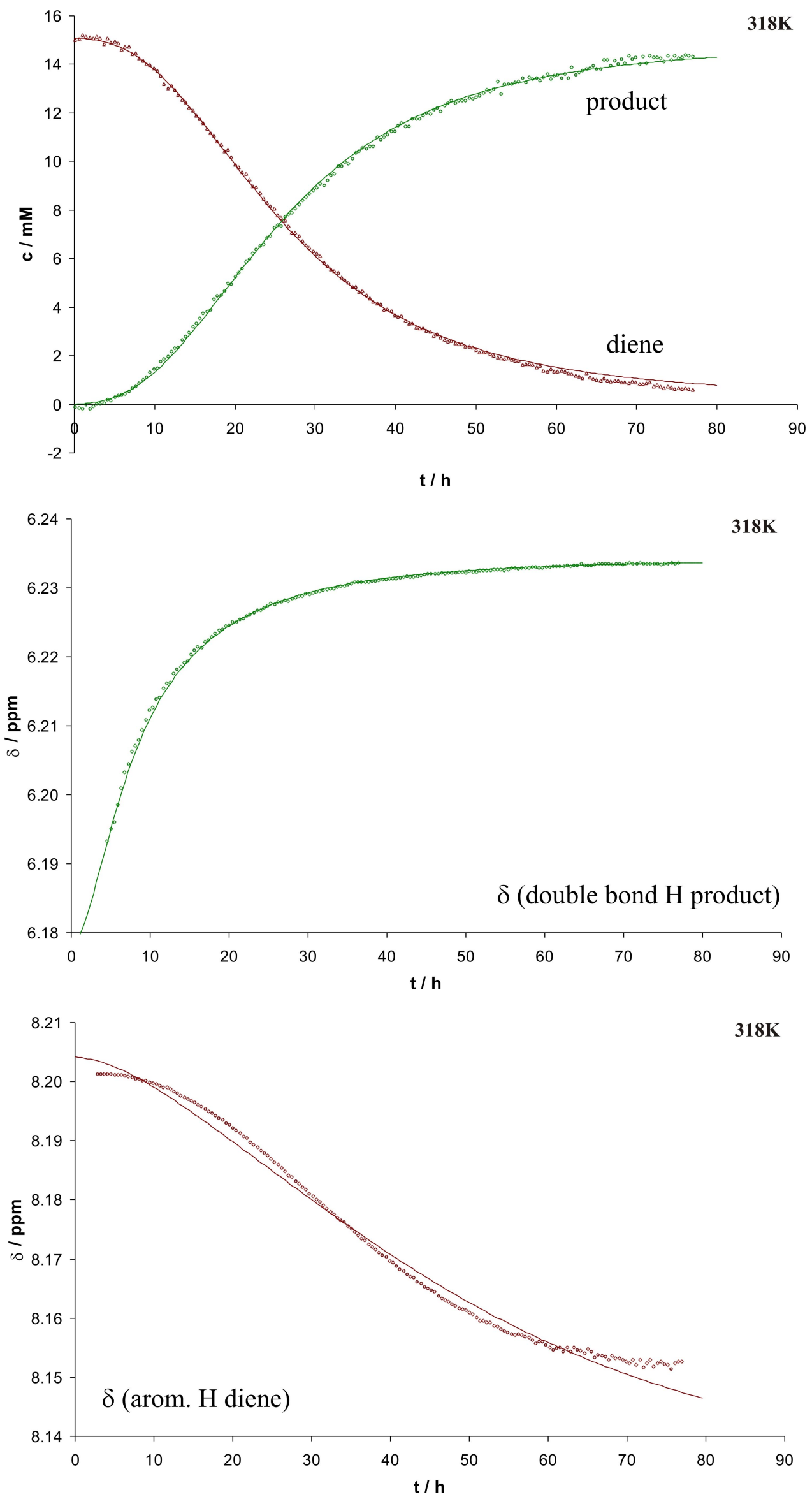

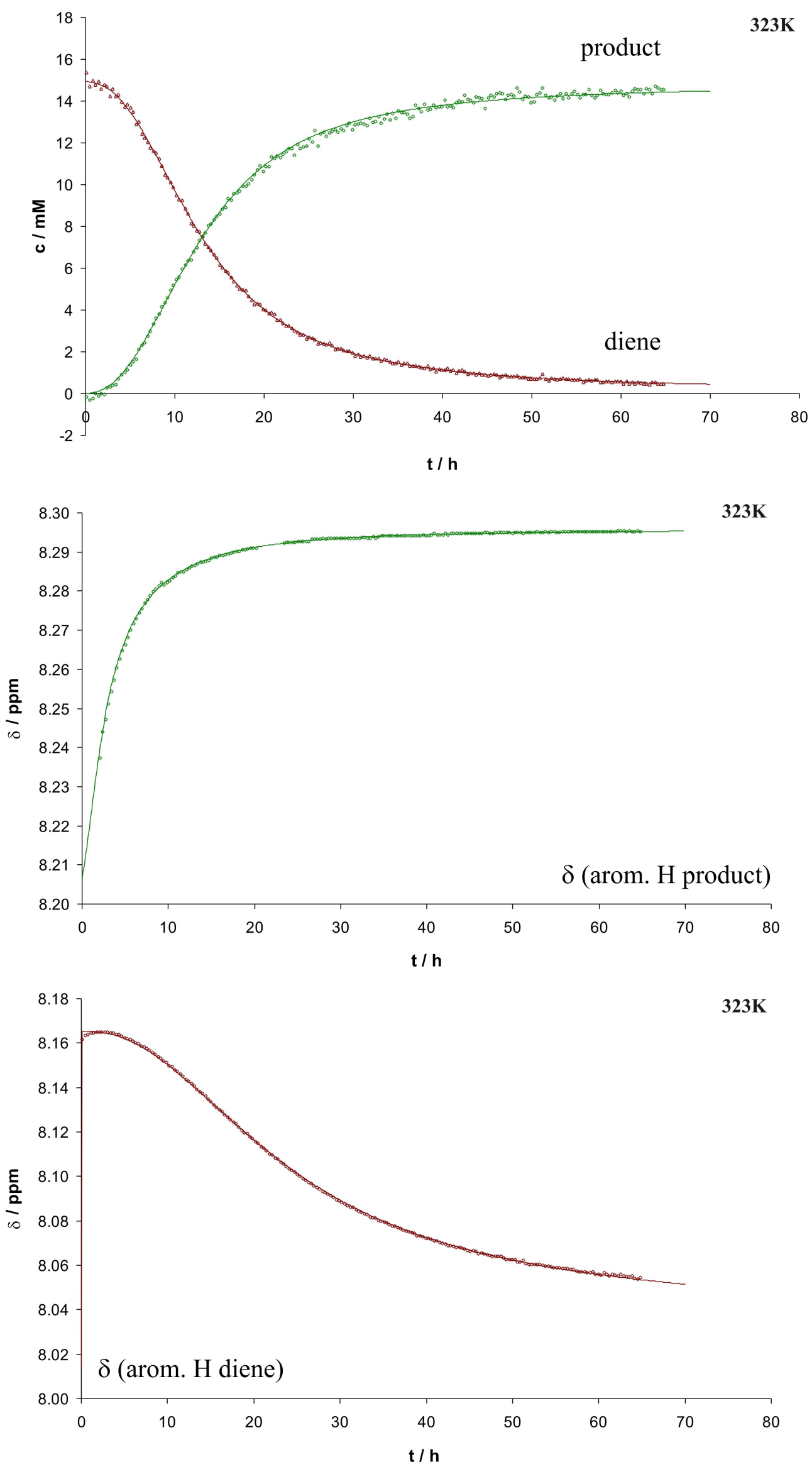

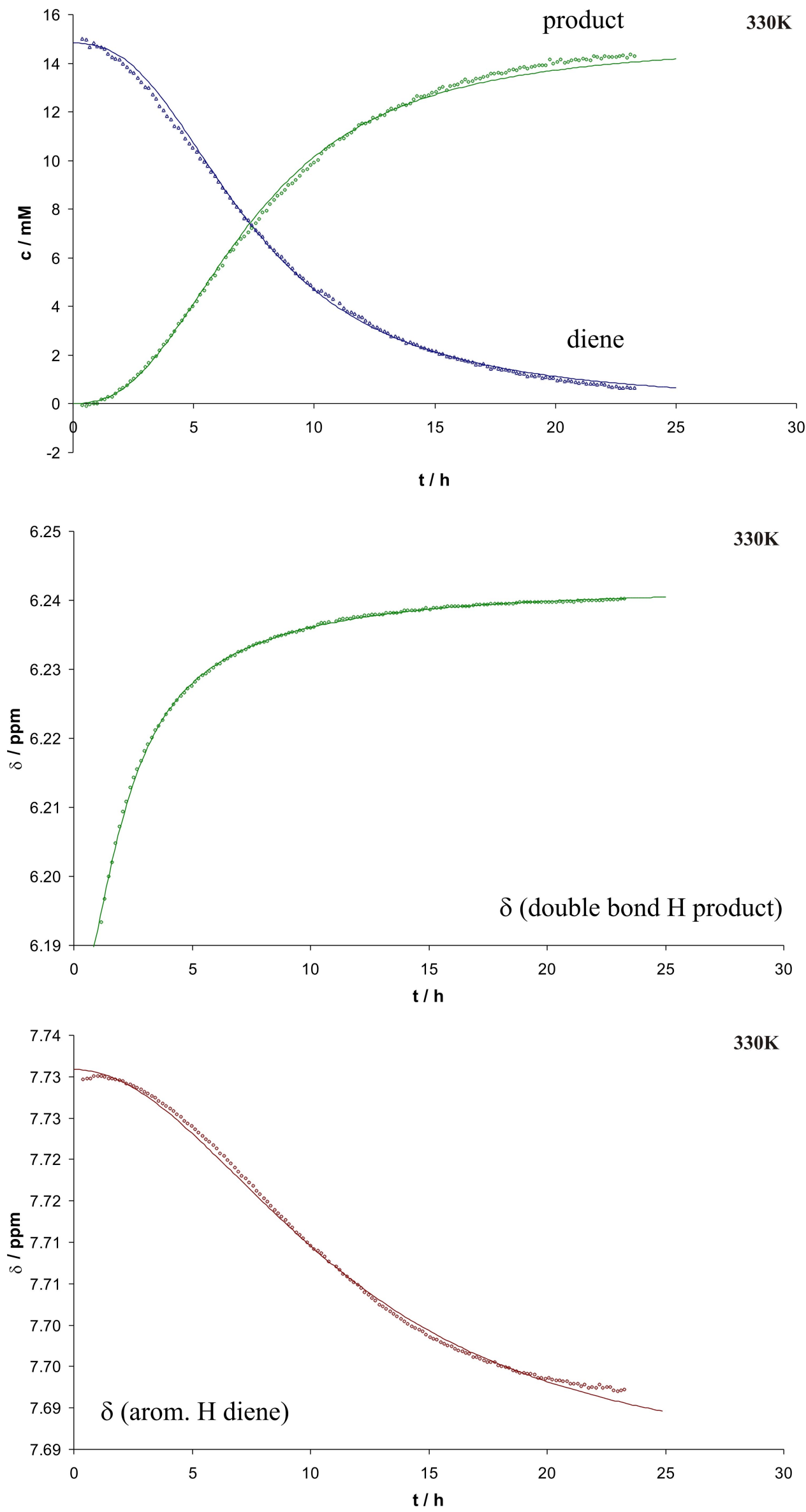

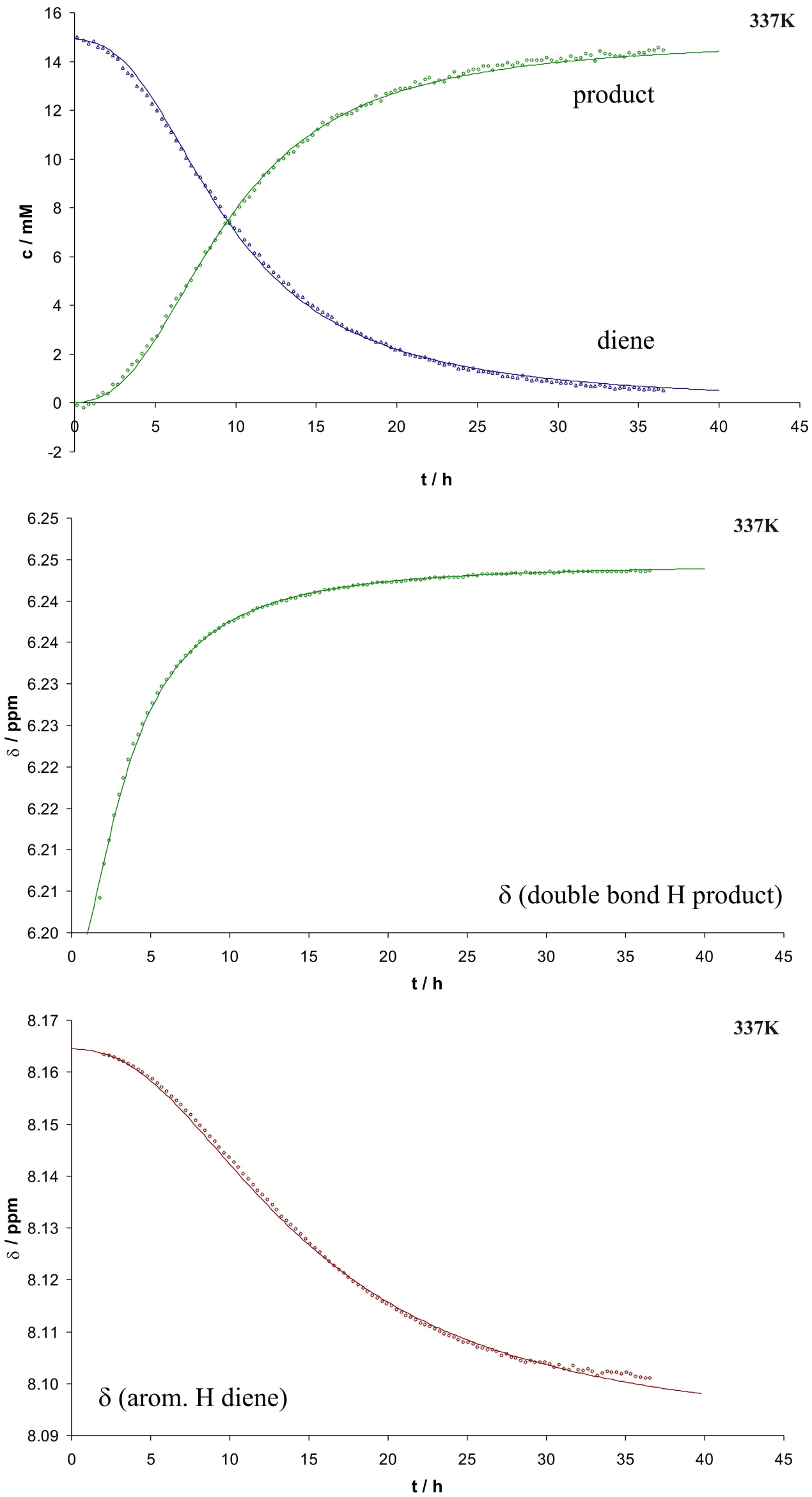

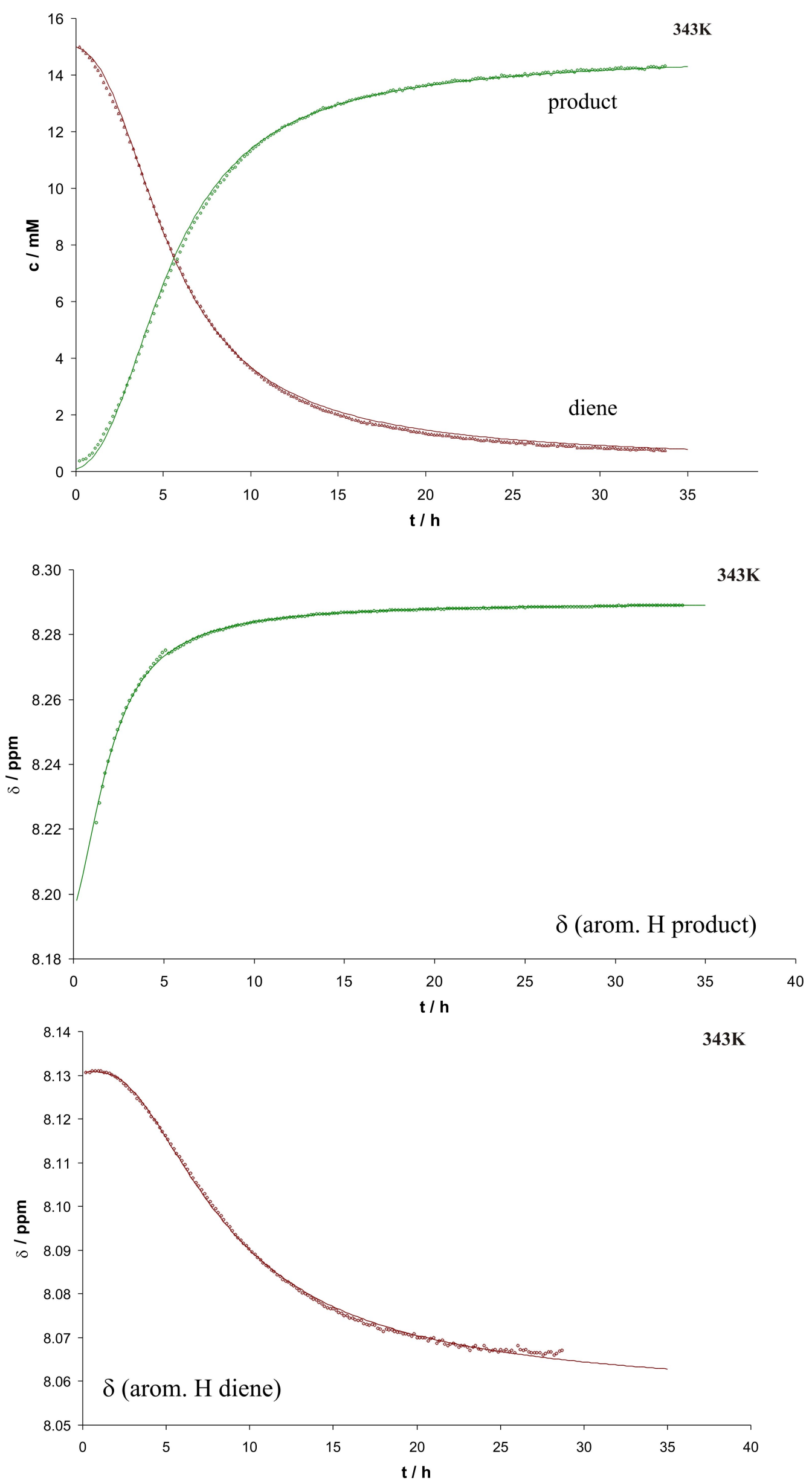
